\title{
Differential Inhibitory Configurations Segregate Frequency Selectivity in the Mouse Inferior Colliculus
}

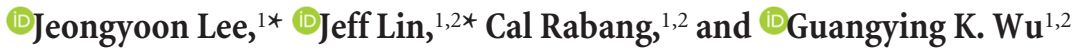 \\ ${ }^{1}$ George Washington Institute for Neuroscience, Department of Psychology, and ${ }^{2}$ Cognitive Neuroscience Program, George Washington University, \\ Washington DC 20052
}

Receptive fields and tuning curves of sensory neurons represent the neural substrates that allow animals to efficiently detect and distinguish external stimuli. They are progressively refined to create diverse sensitivity and selectivity for neurons along ascending central pathways. However, the neural circuitry mechanisms have not been directly determined for such fundamental qualities in relation to sensory neurons' functional organizations, because of the technical difficulty of correlating neurons' input and output. Here, we obtained spike outputs and synaptic inputs from the same neurons within characteristically defined neural ensembles, to determine the synaptic mechanisms driving their diverse frequency selectivity in the mouse inferior colliculus. We find that the synaptic strength and timing of excitatory and inhibitory inputs are configured differently and independently within individual neurons' receptive fields, which segregate sensitive and selective neurons and endow neural populations with broad receptive fields and sharp frequency tuning. By computationally modeling spike outputs from integrating synaptic inputs and comparing them with real spike responses of the same neurons, we show that space-clamping errors did not qualitatively affect the estimation of spike responses derived from synaptic currents in in vivo voltage-clamp recordings. These data suggest that heterogeneous inhibitory circuits coexist locally for a parallel but differentiated representation of incoming signals.

Key words: auditory system; frequency tuning; imbalanced inhibition; in vivo voltage-clamp recording; inferior colliculus

\section{Significance Statement}

Sensitivity and selectivity are functional qualities of sensory systems to facilitate animals' survival. There is little direct evidence for the synaptic basis of neurons' functional variance within neural ensembles. Here we adopted a novel framework to fill such a long-standing gap by uniting population activities with single cells' spike outputs and their synaptic inputs. Furthermore, the effects of space-clamping errors on subcortical synaptic currents were evaluated in vivo, by comparing recorded spike responses and simulated spike outputs from computationally integrating synaptic inputs. Our study illustrated that the synaptic strength and timing of inhibition relative to excitation can be configured differently for neurons within a defined neural ensemble, to segregate their selectivity. It provides new insights into coexisting heterogeneous local circuits.

\section{Introduction}

From peripheral sensory receptors to the neocortex, massive convergent and divergent connections construct individual neurons' sensitivity and selectivity to progressively more complex infor-

\footnotetext{
Received March 23, 2019; revised June 20, 2019; accepted June 30, 2019.

Author contributions: G.K.W. designed research; J. Lee, J. Lin, C.R., and G.K.W. performed research; J. Lee, J. Lin, and G.K.W. contributed unpublished reagents/analytic tools; J. Lee, J. Lin, and G.K.W. analyzed data; G.K.W. wrote the paper.

This work was supported by Grants from the Whitehall Foundation, NARSAD Young Investigator Award from the Brain and Behavior Research Foundation, University Facilitating Fund and startup fund from the George Washington University, and a pilot award from DC-IDDRC program at Children's National Medical Center to G.K.W. We thank Drs. M. T. Colonnese, A.-S. LaMantia, D. O'Halloran's for advice and comments on the paper, and Dr. Paul Marvar for advice on language use and Enago for copyediting.

The authors declare no competing financial interests.

*J. Lee and J. Lin contributed equally to this work.

Correspondence should be addressed to Guangying K. Wu at gwu2@gwu.edu.

https://doi.org/10.1523/JNEUROSCI.0659-19.2019

Copyright $\odot 2019$ the authors
}

mation, which enables animals to efficiently detect and precisely recognize behaviorally significant cues for survival (Kaas, 1989; Popper and Fay, 1992). As neurons are organized into functional subdomains in many sensory systems (DeAngelis et al., 1999; Shepherd and Svoboda, 2005), a few long-standing but fundamental questions are inherent to the operation of local neural circuits: (1) Is the selectivity of neurons gradually distributed into separate streams? (2) Is the selectivity of neurons refined by different patterns of excitatory and inhibitory inputs in a neural ensemble? (3) Are neural populations optimized for selectivity?

In the central auditory system, neurons are selective to a particular range of sound frequency and intensity, as reflected by their frequency-intensity tonal receptive fields (TRFs) and tuning curves (Davies et al., 1954; Kiang et al., 1965; Young and Brownell, 1976). Such selectivity initially originates from inner hair cells in the cochlea (Russell and Sellick, 1977; Dallos, 1985). The central nu- 
cleus of the inferior colliculus (CNIC), a core subdivision of the auditory midbrain, receives all the ascending projections from the brainstem and shapes neurons' responses via heterogeneous sources of excitation and inhibition, before acoustic information is further processed in the medial geniculate body and the auditory cortex (Beyerl, 1978; Winer and Schreiner, 2005). Although auditory neurons have $\mathrm{V}$-shaped spike TRFs, different patterns of spike TRFs were observed in the CNIC, including type I (neurons with a shallower slope on one side of their TRFs), type II (neurons with steep slopes on both sides of their TRFs), type III (neurons with symmetrical slopes), and type IV neurons (neurons with complex or enclosed TRFs; Egorova et al., 2001; Hage and Ehret, 2003). Here we have asked whether different configurations of excitatory and inhibitory inputs could construct individual neurons' selectivity to sound frequency within a characteristically defined neural ensemble.

Previously, excitatory and inhibitory areas of CNIC neurons' TRFs have been derived from spike responses, by either presenting a two-tone inhibition paradigm or blocking GABA receptors (Katsuki et al., 1959; Palombi and Caspary, 1996; LeBeau et al., 2001; Alkhatib et al., 2006). Recently, in vivo whole-cell voltageclamp recordings have been used to directly measure the excitatory and inhibitory synaptic inputs underlying specific functions of CNIC neurons, such as encoding interaural level differences or creating direction selectivity to frequency-modulated sounds (Kuo and Wu, 2012; Xiong et al., 2013; Ono and Oliver, 2014a). However, the impact of spectrotemporal interactions between excitatory and inhibitory inputs on CNIC neurons' diverse selectivity remain unexplored. Moreover, the lack of association between spike activities and synaptic responses from the same neurons challenges the efforts to validate proposed synaptic mechanisms. It is unknown whether other factors such as intrinsic properties of neurons or spaceclamping errors in voltage-clamp recordings would compromise the interpretation of these results and to what extent (Spruston et al., 1993; Priebe and Ferster, 2008).

To validate the significance of synaptic mechanisms for neurons' selectivity, we began with the analysis of neural populations in the CNIC of mice, continued with cell-attached recordings of spike outputs from single cells within them, and finally integrated these data with voltage-clamp recordings of excitatory and inhibitory inputs from the same neurons. To further address the impact of spaceclamping errors in vivo, we compared spike responses from cellattached recordings with those from computational simulations mimicking the potential distortions of synaptic currents. This qualitative evaluation of space-clamping errors clarified how spike outputs reflect the outcome of synaptic mechanisms; a relationship that has not previously been determined in vivo. We found that the strength and timing of excitatory and inhibitory inputs within synaptic TRFs could be differentially configured for nearby neurons, which resulted in their diverse selectivity to incoming stimuli.

\section{Materials and Methods}

\section{Animal model and surgical procedures}

Male and female CBA/CaJ mice (6-15 weeks old and weighing 17-25 g, Jackson Laboratories) were used in this study. All experimental procedures were complied with the National Institutes of Health guidelines and were approved by the Institutional Animal Care and Use Committee of George Washington University.

Mice were anesthetized with ketamine $(100 \mathrm{mg} / \mathrm{kg}$; Ketaject, Henry Schein) and xylazine (10 mg/kg; AnaSed, Henry Schein) or urethane (1 $\mathrm{g} / \mathrm{kg}$ ) and chlorprothixene $(4 \mathrm{mg} / \mathrm{kg})$ via intraperitoneal injections. A tracheotomy was performed, and a glass tube was inserted into the trachea to facilitate breathing during the experiment (Moldestad et al., 2009). A craniotomy was performed above the inferior colliculus con- tralateral to the ear with a customized earphone, and the dura was removed before the entry of electrodes. Animals' body temperature was maintained at $37.5^{\circ} \mathrm{C}$ by a feedback heating system (Harvard Apparatus).

\section{In vivo electrophysiology}

Multiunit activities (MUAs): all recordings were performed in a soundproof booth (Industrial Acoustics). Multiunit spike responses were recorded using parylene-coated tungsten microelectrodes with an impedance of $2 \mathrm{M} \Omega$ (FHC; Wu et al., 2008; Kuo and Wu, 2012). Electrode signals were amplified (Model 1700 differential AC amplifier, AM Systems), and filtered between 300 and $6000 \mathrm{~Hz}$ in a custom-developed software (LabVIEW, National Instrument). Sound was delivered through the earphone inserted into the left ear canal (STAX SR-003). The right ear canal was plugged by clay before the pinna was sutured by surgical clips (Kuo and Wu, 2012; Li et al., 2018). Pure tones $(0.5-64 \mathrm{kHz}$ at 0.1 -octave intervals; 25,50 , or $100 \mathrm{~ms}$ duration; $3 \mathrm{~ms}$ ramp) at eight 10-dB-spaced sound intensities were delivered pseudorandomly. The rate of acoustic stimulation was $\sim 1.8 / \mathrm{s}$, and each tone stimulus was followed by a $500 \mathrm{~ms}$ interstimulus interval. Earphones were calibrated at $70 \mathrm{~dB}$ SPL with a deviation of $\pm 2 \mathrm{~dB}$ SPL for the testing frequency range before experiments (2691-A-0S2, Brüel and Kjær; Kuo and Wu, 2012; Hamilton et al., 2013; Li et al., 2018). Total harmonic distortion was $<1.5 \%$. In this study, pre-mapping by extracellular recordings was always performed to locate the CNIC before cell-attached and whole-cell recordings. The recording tracks were consistently marked by electrodes coated with fluorescent dye DiI, which had no effects on neural responses as shown in previous studies (DiCarlo et al., 1996; Lim and Anderson, 2007; Mauger et al., 2010). The CNIC can be distinguished from the other two major subdivisions (dorsal cortex and external nucleus of the inferior colliculus) by its stereotactic position and its physiological properties (Clopton and Winfield, 1973; Aitkin et al., 1994; Malmierca et al., 2008; Fig. $1 A, B)$. After electrophysiological recordings, animals received an overdose of ketamine and xylazine before transcardially perfused with $4 \%$ paraformaldehyde. Their brains were sectioned at $50 \mu \mathrm{m}$ transversely, and then examined under the fluorescent microscope and imaged using an AMSCOPE MD600E camera.

Cell-attached recordings: recordings were made with AxoPatch 200B (Molecular Devices). Neurons were targeted within 300-1200 $\mu \mathrm{m}$ of depths below the surface of the midbrain. The skull opening was covered with $4-5 \%$ agarose to prevent brain pulsation during recordings. The glass pipette $(\sim 7 \mathrm{M} \Omega)$ was loaded with intracellular solution for voltageclamp recordings (in $\mathrm{mM}$ ): 125 Cs-gluconate, 5 TEA-Cl, 4 MgATP, 0.3 GTP, 10 phosphocreatine, 10 HEPES, 0.5 EGTA, and 2 CsCl. The pH value was adjusted to 7.25 and the osmolarity to $295-305 \mathrm{~mm}$. QX-314 (2.5 mM) was included to improve the voltage-clamping of cell's membrane (Nelson et al., 1994). Under the voltage-clamp mode, a holding potential of $-40 \mathrm{mV}$ was used to monitor the change of resistance and currents in the circuit. The formation of a loose-seal between the pipette tip and cell's membrane was indicated when the resistance reached $0.2-1$ $\mathrm{G} \Omega$. It allowed an isolation of spikes only from the patched cell. Then, recording was performed with the holding voltage switched off. Spike responses were reflected by the current spikes. Signals were filtered at 0.1-10 kHz. Spike waveforms were determined off-line using the custom-developed LabVIEW software.

Whole-cell recordings were made after successful cell-attached recordings (Wu et al., 2008; Poo and Isaacson, 2009; Li et al., 2018). To break the cell membrane, a series of pulses with negative pressure were applied to the cell by a syringe connected to the glass pipette, when voltage pulses were monitored to detect the formation of whole-cell configurations (Margrie et al., 2002). The pipette and cell membrane capacitances were completely compensated, and the series resistance (25-45 $\mathrm{M} \Omega$ ) was compensated by $50-60 \%$, so that an effective series resistance of 15-25 $\mathrm{M} \Omega$ can be achieved. Neurons were considered for further analyses if their recordings showed stable capacitance and resistance, and if their resting membrane potentials were between -65 and $-55 \mathrm{mV}$, according to previous studies of neurons' membrane properties in mouse inferior colliculus (Tan et al., 2007). To obtain synaptic inputs, neurons were clamped at -70 and $0 \mathrm{mV}$, which respectively correspond to the reversal potentials of inhibitory and excitatory currents, as de- 
A

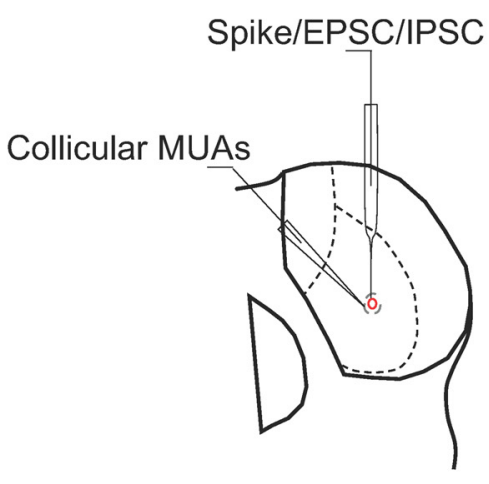

C

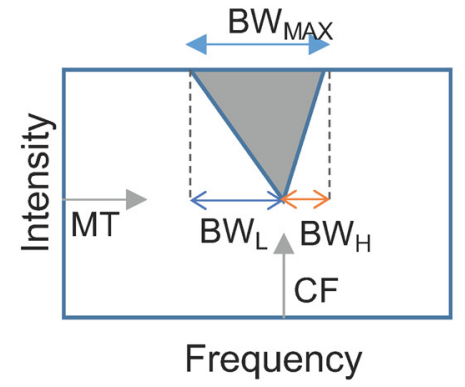

E

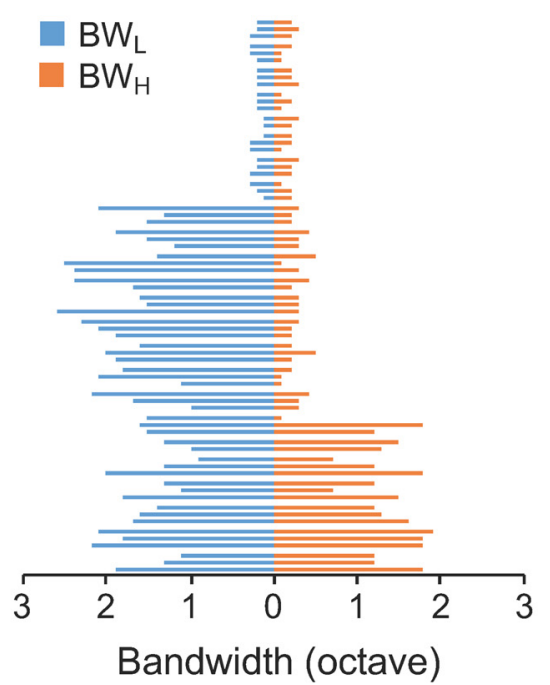

B
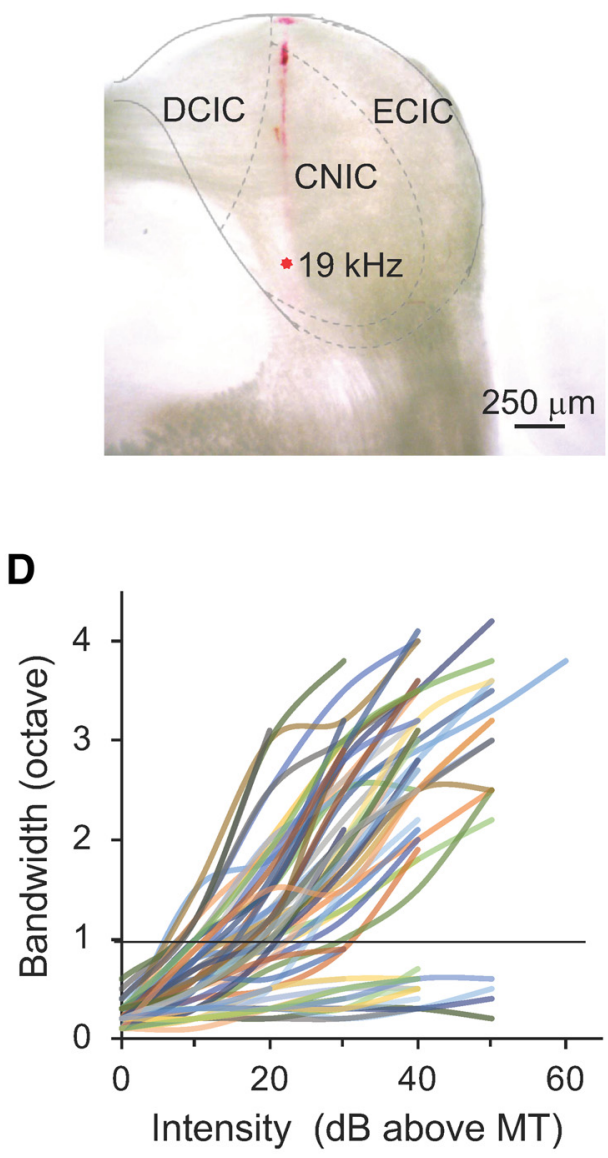

$\mathbf{F}$

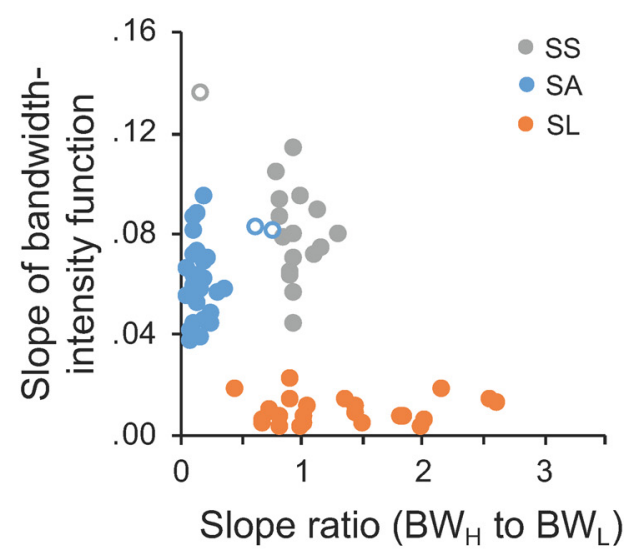

Figure 1. Multimodal recordings in the mouse CNIC and cell-type categorization. $A$, A schematic drawing of multimodal recordings in the mouse CNIC with extracellular recording of MUAs, cell-attached recording of single cells' spikes, and voltage-clamp recording of excitatory postsynaptic currents (EPSCs) and inhibitory postsynaptic currents (IPSCs). $\boldsymbol{B}$, An example recording track labeled in Dil. Dashed lines delineate the boundaries of subdivisions in the mouse CNIC similar to A. C, A schematic TRF of CNIC neurons in the sound frequency and intensity domain. D, Frequency range (bandwidth) as a function of intensity above MT for all single neurons from cell-attached recordings $(n=69)$. Horizontal line, one octave. $E, B W_{L}(b l u e)$ and $B W_{H}$ (orange) for all the individual cells $(n=69) . \boldsymbol{F}$, Cluster analysis of single cells. Slope of bandwidth-intensity function indicates the slope of linear regression of frequency range on sound intensity above MT from individual traces in $\boldsymbol{D}$. Slope ratio $\left(\mathrm{BW}_{\mathrm{H}}\right.$ to $\left.\mathrm{BW}_{\mathrm{L}}\right)$ were obtained from $\boldsymbol{E}$. Solid dots: Neurons classified consistently by both $k$-means clustering and eyeball sorting; open dots: inconsistently-classified neurons.

scribed in our previous studies (Wu et al., 2008; Zhou et al., 2010; Kuo and $\mathrm{Wu}, 2012)$.

\section{Computational modeling}

To further examine the contribution of excitatory and inhibitory inputs to the spike outputs, a single-compartment neuron model was used to simulate membrane potential responses from synaptic inputs. The excit- atory and inhibitory synaptic inputs to such a neuron model were simulated by the following equation (Zhou et al., 2010):

$$
I(t)=a * H\left(t-t_{0}\right) *\left(1-e^{-\left(t-t_{0}\right) /\left(\tau_{\text {rise }}\right)}\right) * e^{-\left(t-t_{0}\right) /\left(\tau_{\text {decay }}\right)},
$$

where $I(t)$ is synaptic input, $a$ is the factor to determine the amplitude of synaptic inputs, $H(t)$ is a Heaviside step function, $t_{0}$ is the onset delay of 
the input, $\tau_{\text {rise }}$ is the time constant for the rising phase, and $\tau_{\text {decay }}$ is the time constant for the decay phase. These parameters were chosen by fitting the averaged shape of recorded synaptic currents in our experiment.

Membrane potential changes were simulated from the modeled synaptic inputs. An integrate-and-fire model was used as previously described (Somers et al., 1995; Wehr and Zador, 2003; Zhou et al., 2010; Kuo and $\mathrm{Wu}, 2012)$ :

$$
\begin{aligned}
V m(t+d t)=-\frac{d t}{C}[G e(t) *( & V m(t)-E e)+G i(t) *(V m(t) \\
& -E i)+G r(V m(t)-E r)]+V m(t),
\end{aligned}
$$

where $\operatorname{Vm}(t)$ is the membrane potential at $t, C$ is the whole-cell capacitance, and $E \mathrm{r}$ is the resting membrane potential $(-55 \mathrm{mV})$. The parameters of $C$ and $E r$ were based on our experimental data. Gr, the resting conductance, was calculated by $G r=C \times G \mathrm{~m} / \mathrm{Cm}$, where $\mathrm{Gm}$ is the specific membrane conductance set to $2 \mathrm{e}^{-5} \mathrm{~S} / \mathrm{cm}^{2}$ and $C \mathrm{~m}$ is the specific membrane capacitance set to $1 \mathrm{e}^{-6} \mathrm{~F} / \mathrm{cm}^{2}$ (Hines, 1993). Ge and Gi, excitatory and inhibitory synaptic conductance, were derived from the equation of $I(t, V)=G_{\mathrm{r}}\left(V-E_{\mathrm{r}}\right)+G_{\mathrm{e}}(t)\left(V-E_{\mathrm{e}}\right)+G_{\mathrm{i}}(t)\left(V-E_{\mathrm{i}}\right)$ as previously reported (Anderson et al., 2000; Wehr and Zador, 2003; Kuo and $\mathrm{Wu}, 2012) . I$ is the synaptic current at the time point of $t ; V$ is the holding voltage respectively set to the reversal potentials of excitatory or inhibitory currents. $E_{\mathrm{e}}(0 \mathrm{mV})$ is the reversal potential of excitatory currents mediated by glutamate receptors. Glycine and GABA are both inhibitory neurotransmitters in the CNIC (Caspary et al., 1990; Koch and Grothe, 1998). A recent study suggests that co-released GABAergic and glycinergic currents are similar in synaptic signaling and interchangeable for maintaining an inhibitory homeostasis (Moore and Trussell, 2017). Thus, $E_{\mathrm{i}}(-70 \mathrm{mV})$ is close to the reversal potential of inhibitory currents mediated by $\mathrm{GABA}_{\mathrm{A}}$ and glycinergic receptors (Kuo and $\mathrm{Wu}, 2012$; Ono and Oliver, 2014a). $V(t)$, the actual clamping voltage, was corrected by $V(t)=V_{\mathrm{h}}-R_{\mathrm{s}} \times I(t)$, where $R_{\mathrm{s}}$ is the compensated series resistance, and $V_{\mathrm{h}}$ is the holding voltage set by the amplifier. The junction potential $(\sim 12 \mathrm{mV})$ was also corrected. By holding the recorded cell at $E_{\mathrm{i}}$ and $E_{\mathrm{e}}, G_{\mathrm{e}}$ and $G_{i}$ were computed, reflecting pure excitatory and inhibitory synaptic inputs. Activation of NMDA receptors can be ignored when the cell is clamped at $-70 \mathrm{mV}$ (Hestrin et al., 1990; Jahr and Stevens, 1990; Pinault, 1996). Thus, the evoked synaptic currents are primarily mediated by AMPA and $\mathrm{GABA}_{\mathrm{A}}$ receptors.

\section{Experimental design and statistical analyses}

Analysis of TRFs, tuning curve, and synaptic onset timing. Raw data were analyzed using custom-developed programs in MATLAB. TRFs of multiunit recordings were constructed from spike responses with amplitudes exceeding two times the SD of the baseline noise in a $25 \mathrm{~ms}$ time window before the onset of tone stimuli. The maximum number of spikes evoked within a TRF was used as reference to create iso-response curves. Minimum threshold (MT) was determined as the lowest intensity that can evoke spike responses within a TRF or iso-response curve; characteristic frequency $(\mathrm{CF})$ was defined as the frequency at which MT could elicit responses (Fig. 1C).

From cell-attached recordings, single cells were classified into three groups based on the shape of their TRFs in reference to previous studies (Egorova et al., 2001; Hage and Ehret, 2003): (1) the slope of linear regression of frequency range on sound intensities above MT (Fig. 1D); (2) the asymmetry of TRF measured by the ratio of TRF's slope along the high-frequency boundary $\left(\mathrm{BW}_{\mathrm{H}}\right)$ to that along the low-frequency boundary $\left(\mathrm{BW}_{\mathrm{L}}\right.$; Fig. $\left.1 E\right)$; and (3) the maximum frequency range at the highest testing intensity $\left(\mathrm{BW}_{\mathrm{MAX}} ; \mathrm{Fig}\right.$. $\left.1 C\right)$. Three types of neurons were defined and observed in all the animals used in our study. Sensitivesymmetrical (SS) neurons have symmetrical spike TRFs, and their frequency range is sensitive to the change of sound intensity (e.g., steep slopes of linear regression of their frequency range on sound intensity). Sensitive-asymmetrical (SA) neurons have asymmetrical spike TRFs, and their frequency range is also sensitive to the change of sound intensity. Selective (SL) neurons have ultra-sharp spike TRFs with $\mathrm{BW}_{\mathrm{MAX}}<1$ octave, and their frequency range is less sensitive to the change of sound intensity (e.g., flat slopes of linear regression of their frequency range on sound intensity). We performed cluster analysis by $k$-means clustering and eyeball sorting. The classification was consistent for SL neurons by $k$-means clustering and eyeball sorting, and only 3 of 47 sensitive neurons (SS or SA) were inconsistent and excluded from further analyses (Fig. $1 F$ ). The individual cells in this study included $17 \mathrm{SS}, 26 \mathrm{SA}$, and $23 \mathrm{SL}$ neurons.

To compare the TRFs of neurons with different CFs, the sharpness of TRFs was quantified by the quality factors at different intensity levels as $\mathrm{Q}$ values. The equation of $Q=f_{\mathrm{c}} / \Delta f$ was used, where $f_{\mathrm{c}}$ is the $\mathrm{CF}$ and $\Delta f$ is the frequency bandwidth. For example, Q30 values represent the quality factors at the intensity of $30 \mathrm{~dB}$ above MT.

Frequency tuning curves of spike or synaptic responses were constructed by the number of spikes or the peak amplitude of synaptic currents evoked by tones at the same sound intensity. To quantify the balance between excitatory and inhibitory inputs, we calculated the ratio of excitatory frequency range to inhibitory frequency range (E/I ratio), and the ratio of frequency bandwidth at $50 \%$ peak of excitatory tuning curve to that of inhibitory tuning curve $\left(\mathrm{E} / \mathrm{I} \mathrm{ratio}_{50}\right)$. If both ratios are close to one, it suggests that excitatory and inhibitory inputs are approximately balanced (Wu et al., 2008).

The onset latency of excitatory and inhibitory inputs was defined as the time point at which the absolute amplitude of evoked inputs became larger than three times the SD of the baseline fluctuation in a $50 \mathrm{~ms}$ time window before the onset of sound stimulation (Wu et al., 2006).

Assessing the effects of space-clamping errors on spike TRFs. Potential deviations of synaptic currents because of space-clamping errors and cable attenuation at the distal dendrites of recorded neurons should be noted in voltage-clamp recordings, although it has been extensively discussed in recent studies (Spruston et al., 1993; Wehr and Zador, 2003; Tan et al., 2004; Williams and Mitchell, 2008; Beaulieu-Laroche and Harnett, 2018). In our study, we proposed a framework that allowed a qualitative assessment of potential space-clamping error's effects on the analyses of synaptic mechanisms in vivo. First, the sensitivity of the computational model to the hypothetically distorted synaptic currents were evaluated by attenuating the amplitude (10-90\%) of excitatory and inhibitory inputs and adjusting their relative latency between $-8 \mathrm{~ms}$ (inhibition leading) and $8 \mathrm{~ms}$ (excitation leading). Second, the predicted spike TRFs from computational simulations were compared with the real spike TRFs from cell-attached recordings to assess false-positive or falsenegative detection of spike responses. Other analyses, including the linearity of $I-V$ curves ( $I$, evoked synaptic currents, as a function of $V$, holding potentials), also suggest that cells were reasonably clamped, and the synaptic currents were not strongly affected by the nonlinearities of the neurons (Wehr and Zador, 2003; Wu et al., 2008; Kuo and Wu, 2012). This was further indicated by the fact that no obvious excitatory currents were observed when cells were clamped at $0 \mathrm{mV}$. This may be attributed to the blockade of most voltage-dependent currents by cesium, TEA, and QX-314 in the intracellular solution, which reduce the membrane permeability and thus decrease the cable attenuation (Spruston et al., 1993). The relative accuracy of derived excitatory reversal potential $(0 \pm 6 \mathrm{mV})$ also suggests a reasonable voltage-clamping of CNIC neurons, because space-clamping errors would result in apparent deviations from the actual reversal potential (Shu et al., 2003).

\section{Statistical analysis}

Statistically significant differences between conditions were determined using standard parametric or nonparametric tests in MATLAB, SPSS 25.0 and Excel. One-way ANOVA with Tukey post hoc test and $t$ test were used to compare the means of different groups, as detailed in the results. $n$ Values in Figures 1, 2, 4, 6, and 9 refer to the number of cells or recording sites. Experiments were performed from the blind recordings. The experimenter performing recordings was not involved in the off-line data analysis. Sample sizes were not predetermined by statistical methods but were based on those commonly used in the field. The data that support the findings of this study are available from the corresponding author upon reasonable request. 


\section{Results}

Diverse frequency tunings of CNIC neural populations with similar broad spike TRFs

To examine the sensitivity and selectivity of neural populations in the CNIC of mice, we obtained MUAs and mapped spike TRFs in the frequency-intensity domain (Fig. 2). The CNIC was determined by anatomical coordinates, recording tracks, and electrophysiological properties including reliable tone-evoked spike responses within TRFs and a tonotopic organization along the dorsolateral-to-ventromedial axis (Figs. $1 A, B, 2 A$ ). When the recording electrodes were advanced into deeper locations, spike TRFs of MUAs quickly expanded and their CFs increased (Fig. $2 A-C)$. To examine the selectivity of neural populations defined by their CFs, we delineated two-dimensional iso-response contours according to $25,50,75$, and $100 \%$ of the maximal number of spikes, in addition to the contours of spike TRFs (Fig. 2B). Although the change of CFs measured from spike TRFs was less than one octave $(8-16 \mathrm{kHz})$ along this recording track, those measured from $50 \%$ iso-response contours varied by $>2.5$ octaves (e.g., 4-28 kHz; Fig. 2C). A bidirectional shift of CFs was noted: the CFs of $50 \%$ iso-response contours shifted toward lower frequency side for neural populations with low CFs of spike TRFs, whereas they shifted toward higher frequency side for neural populations with high CFs of spike TRFs (Fig. 2C). It is also worth noting that the peaks of neural populations' frequency tuning curves were sequentially selective to different frequencies with varying sharpness, although their frequency ranges largely overlapped for recordings made between 650 and 1600 $\mu \mathrm{m}$ of depths under the surface of the midbrain (Fig. 2D). MT decreased from 20 to $10 \mathrm{~dB}$ SPL, then remained at $20 \mathrm{~dB}$ SPL along the same recording axis (Fig. $2 E$ ). This result suggests that neural populations with similar TRFs could possess different frequency selectivity.

We next analyzed the spike TRFs and frequency tuning of neural populations from 156 recording sites in 11 animals. Q30 values (quality factor measured at $30 \mathrm{~dB}$ above MT to evaluate the sharpness of neurons' frequency representation; see Materials and Methods) and MTs of neural populations characterized by CF were compared (Fig. 2F, G). Q30 of spike TRFs was not significantly different for neural populations with different CFs (one-way ANOVA, $F_{(5,102)}=1.39, p=0.2339$ ). However, Q30 measured from $50 \%$ iso-response contours showed that neural populations with CFs between 8 and $22 \mathrm{kHz}$ had larger values (Fig. $2 F$; one-way ANOVA: $F_{(7,122)}=4.42, p=0.0002$; Tukey post hoc test: $8-11.3$ vs $4-5.6 \mathrm{kHz}, p=0.0157 ; 8-11.3$ vs $5.6-8 \mathrm{kHz}$, $p=0.0433 ; 8-11.3$ vs $11.3-16 \mathrm{kHz}, p=0.9731 ; 8-11.3$ vs $16-$ $22.6 \mathrm{kHz}, p=0.3074 ; 8-11.3$ vs $22.6-32 \mathrm{kHz}, p=0.0070 ; 8-11.3$ vs $32-45.2 \mathrm{kHz}, p=0.0391 ; 8-11.3$ vs $45.2-64 \mathrm{kHz}, p=0.0229)$. In the sound intensity domain (Fig. $2 G$ ), MT of $50 \%$ iso-response contours was lower for neural populations with CFs between 5.6 and $32 \mathrm{kHz}$ (one-way ANOVA, $\mathrm{F}_{7,122)}=5.36, p<0.0001$; Tukey post hoc test: $8-11.3$ vs $4-5.6 \mathrm{kHz}, p=0.0419 ; 8-11.3$ vs $5.6-8$ $\mathrm{kHz}, p=0.1955 ; 8-11.3$ vs $11.3-16 \mathrm{kHz}, p=0.9639 ; 8-11.3 \mathrm{vs}$ $16-22.6 \mathrm{kHz}, p=0.9941 ; 8-11.3$ vs $22.6-32 \mathrm{kHz}, p=0.3545$; $8-11.3$ vs $32-45.2 \mathrm{kHz}, p=0.0003 ; 8-11.3$ vs $45.2-64 \mathrm{kHz}, p=$ $0.0299 ; 11.3-16$ vs $32-45.2 \mathrm{kHz}, p=0.0062$ ), although that of spike TRFs was not significantly different for neural populations with different CFs (one-way ANOVA, $F_{(5,102)}=2.13, p=$ $0.0675)$. These results suggest that neural populations with CFs in the middle and high-frequency range have both greater sensitivity and sharper selectivity to incoming stimuli.

\section{Heterogeneous frequency selectivity of single neurons in the CNIC}

Neural population's great sensitivity and sharp selectivity could be explained by a group of neurons having overlapped broad spike TRFs and sharp frequency tuning measured by the number of spikes, or because of a collection of neurons having different sizes of spike TRFs and weak frequency tuning of spikes. To examine the spike TRFs and frequency tuning of single neurons within a neural population, we applied in vivo cell-attached recording in the CNIC while documenting their locations and recording MUAs at the same time (Fig. 3). Nearby neurons in a neural population with a similar CF can be classified into SS, SA, and SL neurons (see Materials and Methods; Figs. $1 C-F, 3 A-C$ ). Sensitive neurons had typical V-shaped spike TRFs with either symmetrical (SS: $26 \%, n=17$; Fig. $3 A$ ) or asymmetrical (SA: $39 \%, n=26$; Fig. $3 B$ ) slopes at the low-frequency and highfrequency boundaries of TRFs. Their frequency bandwidth was sensitive to the change of sound intensity, which was linearly broadened when sound intensity was increased (Figs. 1D, $3 A, B$ ). Selective neurons (SL: $35 \%, n=23$ ) had ultra-sharp spike TRFs with their maximal frequency range often less than one octave even at the highest testing intensity (e.g., 0.3 octaves at $70 \mathrm{~dB}$ SPL; Fig. $3 \mathrm{C}$ ). Their frequency bandwidth was less sensitive to the change of sound intensity, with only small changes in frequency range along the intensity axis (Figs. $1 D$, $3 C)$. All three types of neurons had the number of evoked spikes quickly reaching to a plateau when tone stimuli moved into their TRFs, which prevented them from showing clear frequency tuning, especially for SS neurons (Fig. 3A-C, color maps). For sensitive neurons, their firing patterns showed consistent early responses within their spike TRFs, in contrast to selective neurons (Fig. 3D-F).

We examined the sharpness of individual neurons' frequency representation by Q30 values. The distribution of Q30 values showed a segregation of sensitive and selective neurons in the CNIC of mice (Fig. 4A). Sensitive neurons had a wide range of CFs, but selective neurons were prominent among neurons with higher CFs $(8-30 \mathrm{kHz}$; Fig. $4 B)$. The MTs of SS, SA, and SL neurons were not significantly different (Fig. 4C,D; oneway ANOVA, $\left.F_{(2,63)}=0.66, p=0.5222\right)$. In general, selective neurons had larger $Q$ values at all sound intensities above MT than sensitive neurons, indicating overall stronger selectivity (Fig. $4 E$; one-tail two-sample $t$ test: $\mathrm{MT}+10 \mathrm{~dB}$, SL vs SS: $t_{(38)}$ $=8.21, p<0.0001$, SL vs SA: $t_{(47)}=8.36, p<0.0001$, SS vs SA: $t_{(41)}=0.74, p=0.23 ; \mathrm{MT}+20 \mathrm{~dB}$, SL vs SS: $t_{(38)}=8.51, p<$ 0.0001 , SL vs SA: $t_{(47)}=8.12, p<0.0001$, SS vs SA: $t_{(41)}=2.70$, $p=0.0050 ; \mathrm{MT}+30 \mathrm{~dB}$, SL vs SS: $t_{(38)}=9.36, p<0.0001, \mathrm{SL}$ vs SA: $t_{(47)}=8.73, p<0.0001$, SS vs SA: $t_{(41)}=3.39, p=$ $0.0008 ; \mathrm{MT}+40 \mathrm{~dB}$, SL vs SS: $t_{(29)}=5.82, p<0.0001$, SL vs SA: $t_{(36)}=5.43, p<0.0001$, SS vs SA: $t_{(31)}=3.31, p=0.0011$; $\mathrm{MT}+50 \mathrm{~dB}$, SL vs SS: $t_{(14)}=9.92, p<0.0001$, SL vs SA: $t_{(11)}=$ $6.64, p<0.0001$, SS vs SA: $\left.t_{(15)}=1.15, p=0.1347\right)$. Sensitive neurons had much smaller temporal jitter (measured by SD) of first spike latency within their spike TRFs than selective neurons (Fig. 4F; one-tail two-sample $t$ test: SS vs SL: $t_{(38)}=$ $-3.00, p=0.0024$; SA vs SL: $t_{(47)}=-4.39, p<0.0001$; SS vs SA: $\left.t_{(41)}=0.84, p=0.2039\right)$. Our data suggest that nearby neurons with similar CFs can be differentiated for frequency analysis, which may allow neural populations to effectively detect and distinguish stimuli by different subpopulations at the same time. 
A
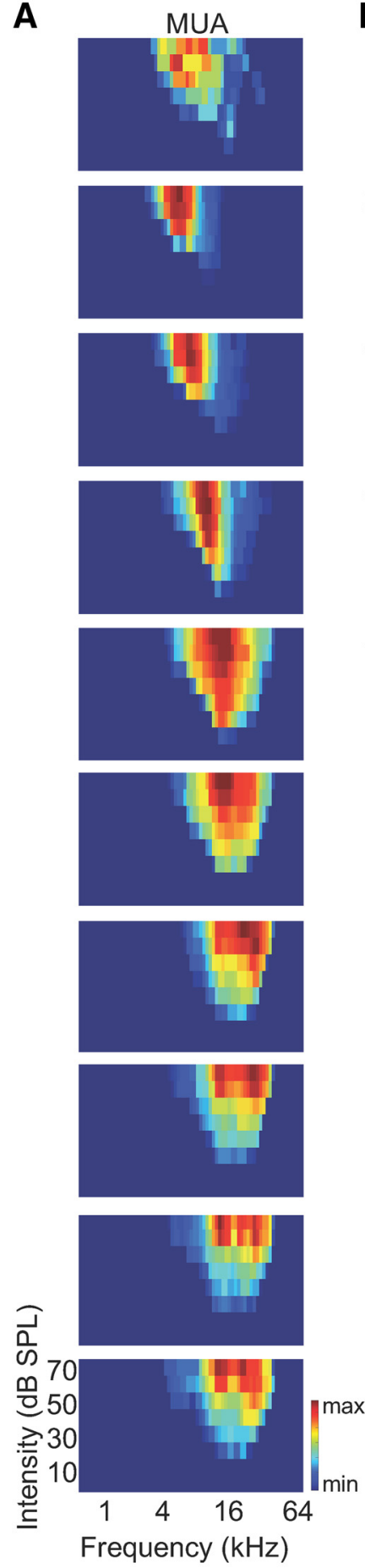

B

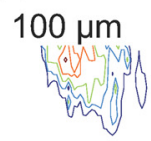

$250 \mu \mathrm{m}$<smiles>[Si]C1C2C=CC1C=C2</smiles>

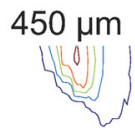

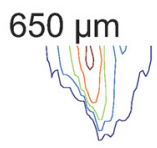

$850 \mu \mathrm{m}$<smiles>c1ccccc1</smiles>

$1050 \mu \mathrm{m}$

$1250 \mu \mathrm{m}$<smiles>c1ccccc1</smiles>

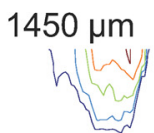

$1600 \mu \mathrm{m}$<smiles>C1CC[AlH]CC1</smiles>

$1800 \mu \mathrm{m}$
C

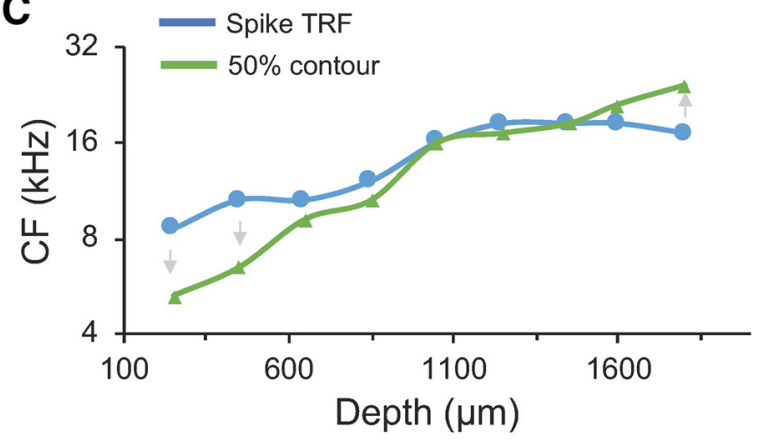

D

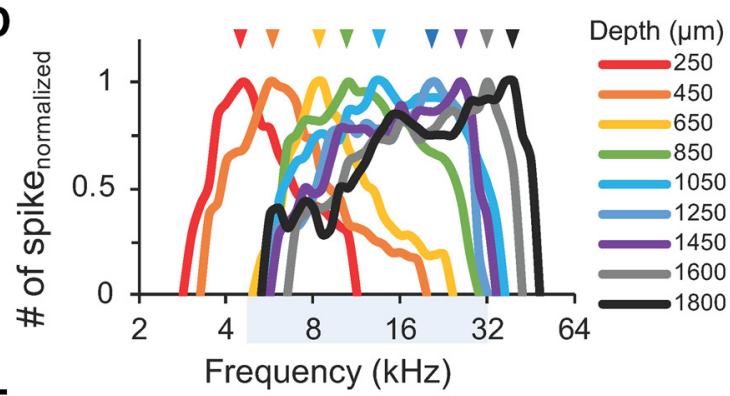

E

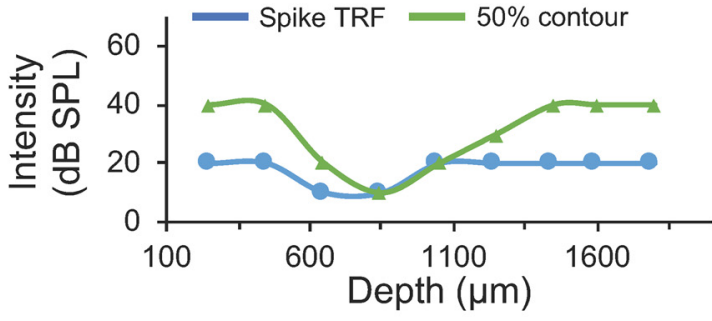

F

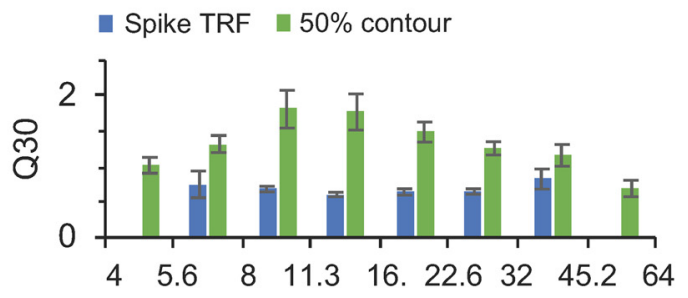

G

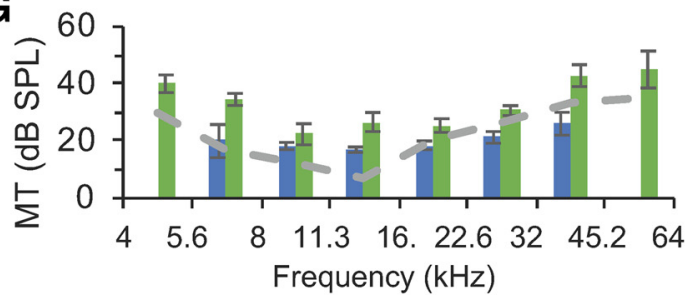

Figure 2. Differential frequency tunings of neural populations with similar broad spike TRFs in the CNIC. $A$, Top to bottom, Color maps of spike TRFs from 10 recording sites along the dorsolateral to ventromedial axis. The boundary of the dorsal cortex of the inferior colliculus and the CNIC can be seen between 100 and $150 \mu \mathrm{m}$ in Figure 1B. Color bar indicates the maximal (top to bottom: 25 , $52,55,56,48,43,47,45,48,39)$ and minimal number (0) of spikes evoked within the TRFs. $B$, Contours of spike TRFs (navy), and iso-response contours of $25 \%$ (blue), $50 \%$ (green), $75 \%$ (orange), and $100 \%$ (red) of maximal spike responses in $A$. C, CFs determined by spike TRFs and $50 \%$ iso-response contours in $\boldsymbol{B}$ along the recording depths. Arrows indicate the directional shift of CFs measured from $50 \%$ iso-response contours and spike TRFs. $\boldsymbol{D}$, Normalized frequency tuning curves at $30 \mathrm{~dB}$ above MT along the recording depths. Arrows indicate the peak responses. Shaded area indicates largely overlapped frequency ranges of neural populations at depths between 650 and $1600 \mu$ m under the pia. $\boldsymbol{E}$, MTs from spike TRFs and 50\% iso-response contours along the recording depths. $\boldsymbol{F}$, Sharpness of frequency range indicated by Q30 values for neural populations with different CFs in 156 multiunit recordings. MUAs were grouped according to their CFs of spike TRFs or $50 \%$ iso-response contours, by dividing testing frequencies $(4-64 \mathrm{kHz})$ into eight groups, with each group covering a frequency range of 0.5 octaves $(4-5.6,5.6-8,8-11.3,11.3-16,16-22.6$, $22.6-32,32-45.2$, and $45.2-64 \mathrm{kHz}$ ). Blue bars indicate the analysis from spike TRFs; green bars indicate the analysis from $50 \%$ iso-response contours. Error bar indicates SEM. G, MTs for neural populations with different (Fs from recordings in $\boldsymbol{F}$. Blue bars indicate the analysis from spike TRFs; green bars indicate the analysis from $50 \%$ iso-response contours; gray dashed curve is the averaged behavioral audiogram between 4 and 64 kHz estimated from previous studies (Ehret, 1974; Zheng et al., 1999; Prosen et al., 2003; Radziwon et al., 2009). Error bars indicate SEM. 
A

B

C

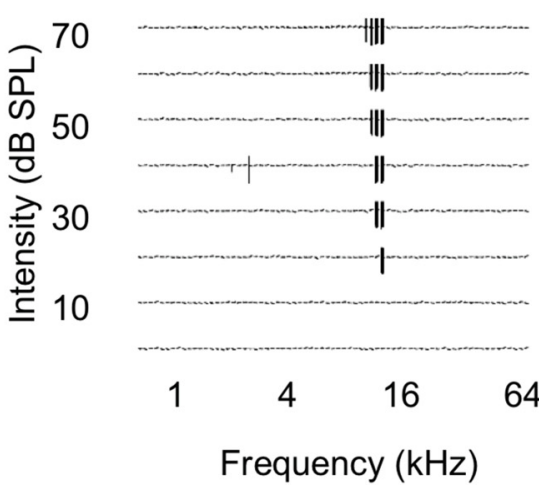

D

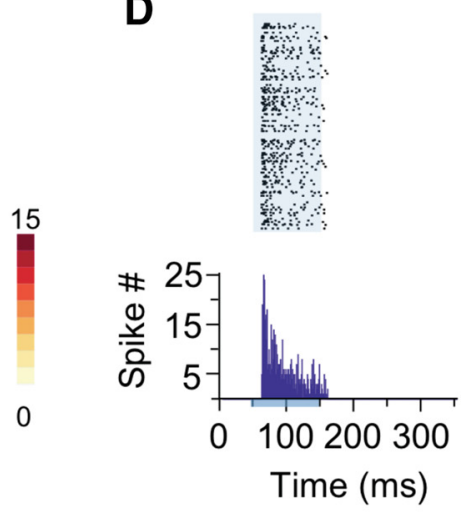

E

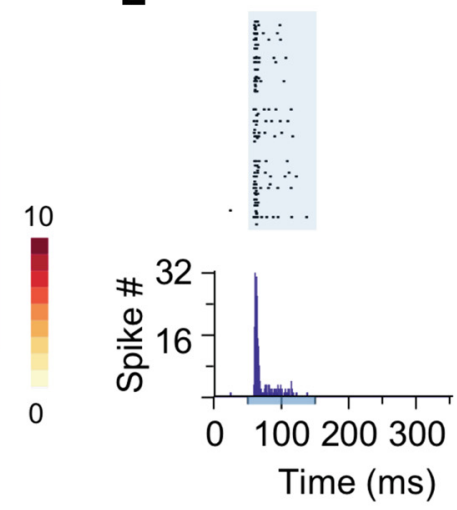

$\mathbf{F}$

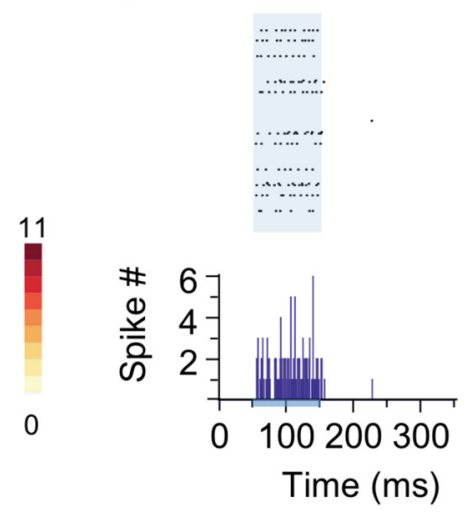

Figure 3. Diverse spike TRFs of single neurons from in vivo cell-attached recordings in the CNIC. $\boldsymbol{A}-\boldsymbol{C}$, Three types of spike TRFs with similar CFs at $16 \mathrm{kHz}$ and locations ( $\sim 50 \mu \mathrm{m}$ distance to each other). Each panel represents 568 individual recording traces of $300 \mathrm{~ms}$ after tone onset; each trace represents a $300 \mathrm{~ms}$ recording of spike response after the onset of a tone stimulus with a particular combination of sound intensity $(0-70 \mathrm{~dB}$ SPL) and frequency $(0.5-64 \mathrm{kHz})$. Blue dashed contour delineates the spike TRFs of multiunit activities near these neurons in $\boldsymbol{A}-\boldsymbol{C}$. Insets, Color maps indicate spike numbers evoked by different tones within TRFs for SS neurons ( $\boldsymbol{A})$, SA neurons $(\boldsymbol{B})$, and SL neurons $(\boldsymbol{C})$. $\boldsymbol{D}-\boldsymbol{F}$, Raster plots and peristimulus time histograms (PSTHs) of the spike responses extracted from recordings in $\boldsymbol{A}$-C. Each raster plots include 568 trials of $350 \mathrm{~ms}$ recordings including a 50 ms time window before the stimulus onset. Shaded areas and bars below $P S T H s$ indicate stimulus onset and duration.

\section{Synaptic mechanisms underlying the divergence of frequency selectivity}

Two possible mechanisms could underlie the differentiated frequency selectivity of CNIC neurons: (1) excitatory synaptic TRFs could have already been constructed differently for sensitive and selective neurons, which largely define their distinct spike TRFs; and (2) excitatory synaptic TRFs could have not yet been segregated for sensitive or selective neurons, however, inhibitory synaptic inputs could play a significant role to achieve the diver- gence. To determine the exact synaptic mechanisms, we dissected the excitatory and inhibitory synaptic inputs of CNIC neurons by in vivo voltage-clamp recordings. For a subset of neurons (neurons in Fig. 3), we were able to obtain voltage-clamp recordings after completing cell-attached recordings $(53 \%, n=35$; SS: $n=$ 12; SA: $n=10$; SL: $n=13$; Fig. 5). Different patterns of excitatory and inhibitory synaptic TRFs were observed from nearby neurons with similar CFs (Fig. 5A-F). For example, the excitatory and inhibitory TRFs were largely matched for sensitive neurons 
A

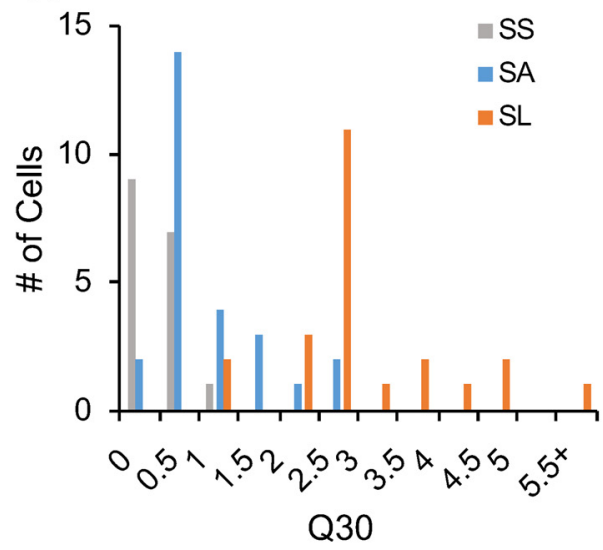

C

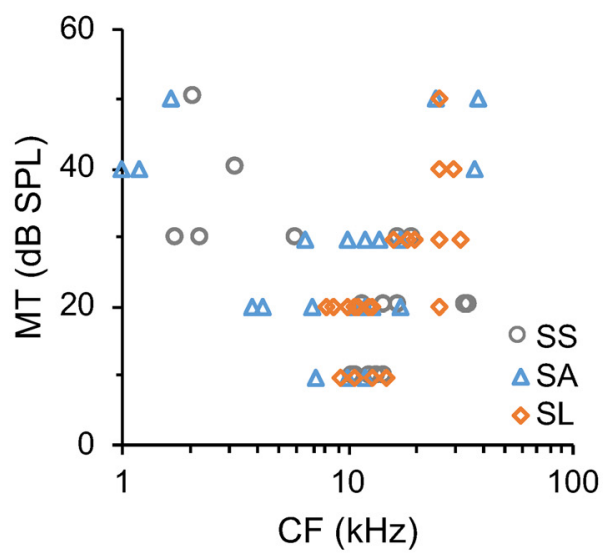

E

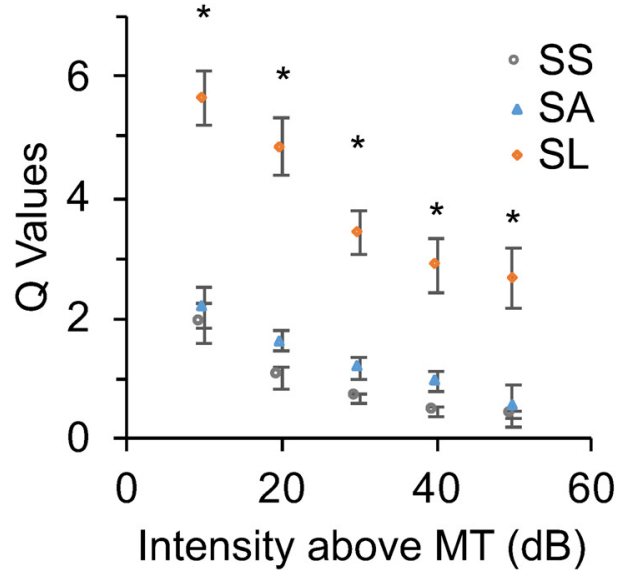

$\mathbf{B}$

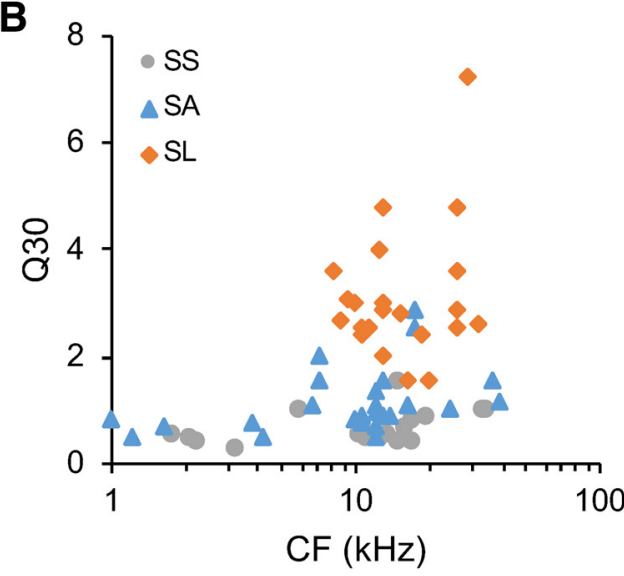

D

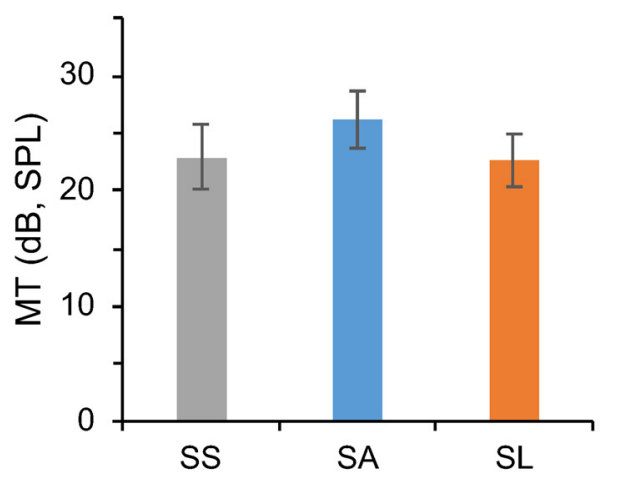

$\mathbf{F}$

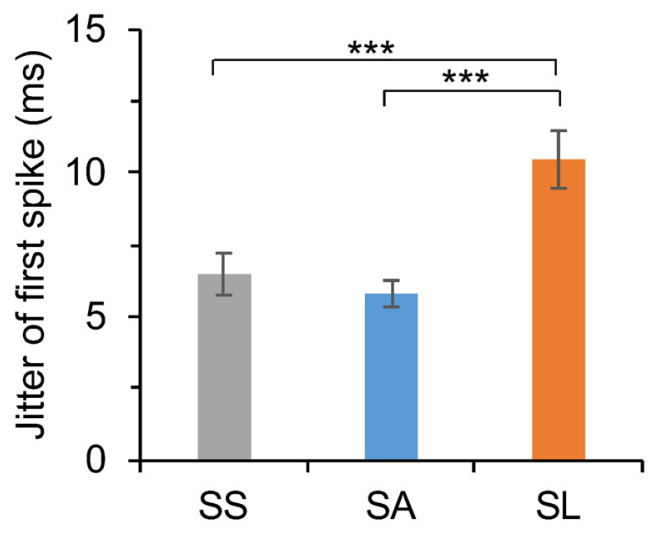

Figure 4. Differential frequency selectivity of single neurons. $A$, Distribution of CNIC neurons with different selectivity measured by Q30 values. SS neurons, Gray; SA neurons, blue; SL neurons, orange. B, Q30 values of major types of neurons versus their CFs. SS, Gray; SA, blue; SL, orange. C, MTs of major types of neurons versus their CFs. D, MTs of major types of neurons (unit in dBSPL, SS: 22.9 $\pm 2.8 ; S A, 26.2 \pm 2.5$; $S L: 22.6 \pm 2.3 ; M \pm S E M) . E, Q$ values for major types of neurons at different sound intensities above MT. Asterisks indicate significant larger $Q$ values of $S L$ neurons. $F$, Temporal jitter measured by the $S D$ of first spike latency within their spike TRFs for three types of neurons (in milliseconds; SS:6.5 \pm 0.7; SA:5.8 $\pm 0.5 ; S L: 10.5 \pm 1.1 ; M \pm S E M$ ). Asterisks indicate significant differences between SL and SS neurons, and between SL and SA neurons.

(Fig. $5 A-D$ ), but not for selective neurons (Fig. $5 E, F)$. Furthermore, three different configurations of frequency tuning curves existed for excitatory and inhibitory inputs: matched excitation and inhibition (Fig. 5G, top, SS neuron), sharp inhibition (Fig. $5 G$, middle, SA neuron), and broad inhibition (Fig. 5G, bottom, SL neuron). For sensitive neurons with asymmetrical TRFs, the peaks of inhibitory frequency tuning curves were shifted and tilted toward the high-frequency side in contrast to those of excitatory frequency tuning curves at all the intensities above their MTs (Fig. 5G, middle).

To further quantify the synaptic selectivity and the balance between excitation and inhibition, we examined the frequency 
A

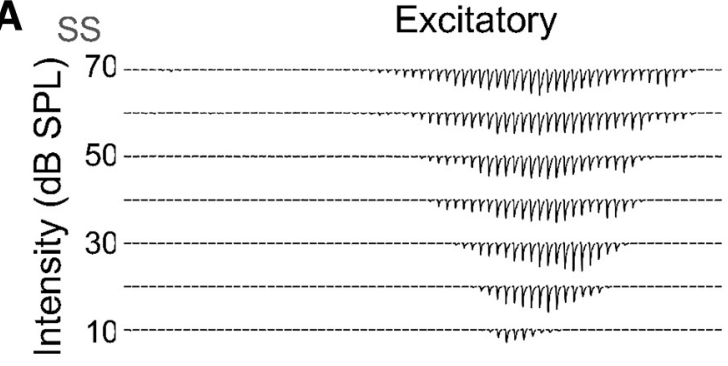

C SA

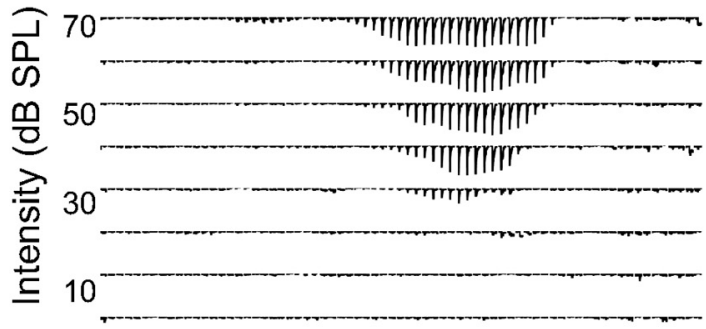

E

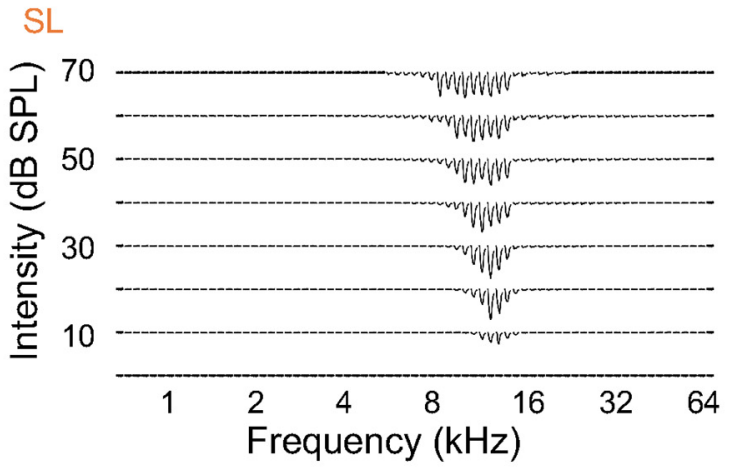

B Inhibitory

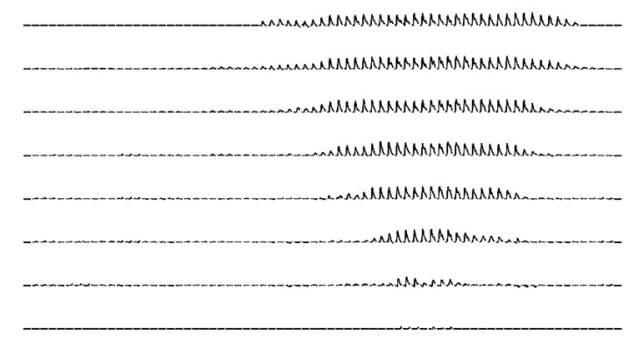

D

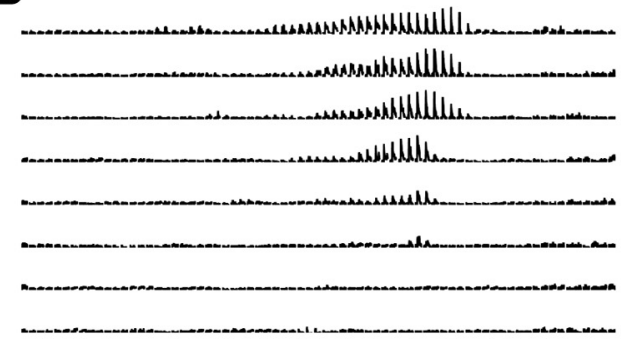

$\mathbf{F}$

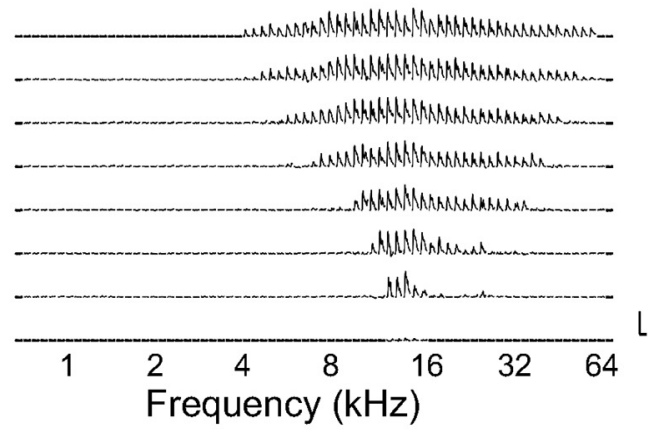

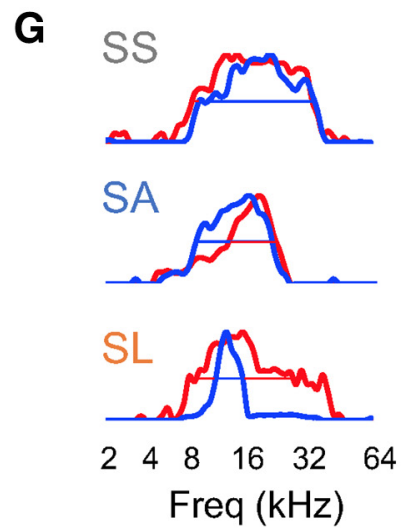
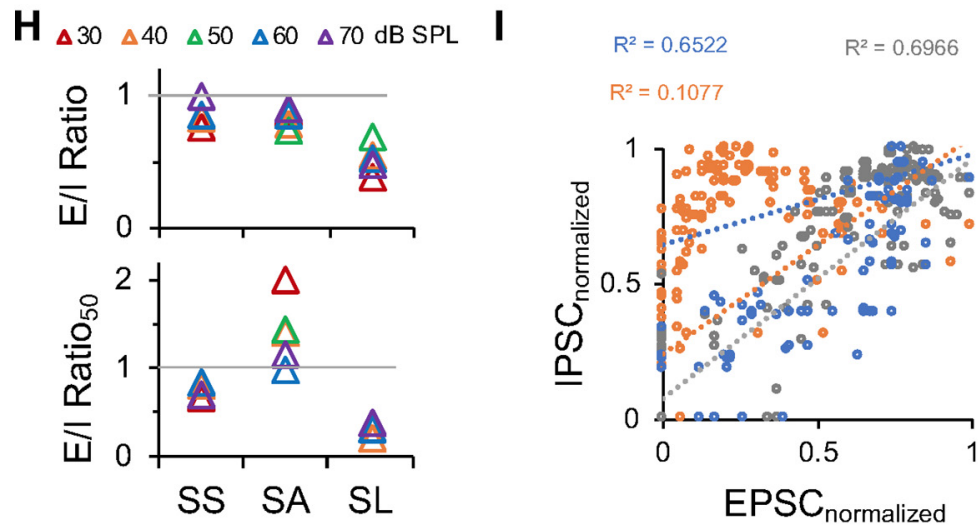

Figure 5. Diverse patterns of excitatory and inhibitory synaptic TRFs obtained from in vivo voltage-clamp recordings. $A, C, E$, Excitatory synaptic TRFs of the three neurons in Figure $3 A-C$. $B, D$, $\boldsymbol{F}$, Inhibitory synaptic TRFs of the neurons in $\boldsymbol{A}, \boldsymbol{C}$, and $\boldsymbol{E}$, respectively. Vertical scale bars: $\boldsymbol{A}, 400 \mathrm{pA} ; \boldsymbol{B}, 270 \mathrm{pA} ; \boldsymbol{C}, 230 \mathrm{pA} ; \boldsymbol{D}, 200 \mathrm{pA} ; \boldsymbol{E}, 370 \mathrm{pA} ; \boldsymbol{F}, 300 \mathrm{pA}$. Horizontal scale bar, $350 \mathrm{~ms}$. $\boldsymbol{G}$, Frequency tuning curves at $30 \mathrm{~dB}$ above MT for the cells in $\boldsymbol{A}-\boldsymbol{F}$. Excitatory inputs show in blue, inhibitory inputs show in red. Straight lines indicate the frequency bandwidths at $50 \%$ of the peak. $\boldsymbol{H}, \boldsymbol{E} / \mathbf{I}$ ratios

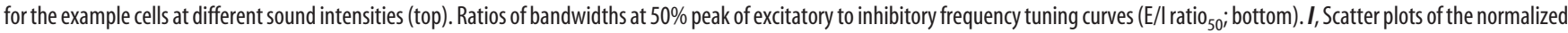
amplitudes of EPSCS (EPSC normalized $_{\text {) }}$ )ersus that of IPSCS (IPSC normalized $_{\text {) }}$ in the synaptic TRFs of the three cells shown in $\boldsymbol{A}-\boldsymbol{F}$ (gray: SS, $n=181 ;$ blue: $S A, n=95 ;$ orange: $S L, n=153$ ). Excitatory and inhibitory synaptic currents were normalized by the maximum amplitude within their respective synaptic TRFs.

range of excitatory and inhibitory inputs, and the frequency bandwidths at $50 \%$ peak of their tuning curves at different sound intensities (Fig. 5G,H). The ratios of excitatory frequency range to inhibitory frequency range (E/I ratio) were close to one for SS and SA neurons, but smaller than one for SL neurons indicating their broader inhibition (Fig. $5 H$, top).
The ratios measured at $50 \%$ peak of frequency tuning curves $\left(\mathrm{E} / \mathrm{I} \mathrm{ratio}_{50}\right)$ were close to one for SS neurons and smaller than one for SL neurons, whereas greater than one for SA neurons indicating their shaper inhibition (Fig. $5 \mathrm{H}$, bottom). Both SS and SA neurons also showed well correlated amplitudes of excitatory and inhibitory inputs evoked by various tones, in- 
stead of the polynomial-like relationship between them for SL neurons (Fig. 5I).

To examine whether synaptic TRFs showed differentiated selectivity similar to spike TRFs, we quantified the sharpness of excitatory and inhibitory TRFs by Q30 values. The distribution of Q30 values indicated different sizes of synaptic TRFs (Q30 of excitatory vs inhibitory inputs, $0.63 \pm 0.19$ vs $0.54 \pm 0.20$, $\mathrm{M} \pm \mathrm{SD}$; one-tail paired $t$ test, $\left.t_{(34)}=2.15, p=0.0192\right)$, but neither excitatory nor inhibitory TRFs showed differentiated selectivity like in Figure 4A (Fig. 6A). The Q values at various intensities indicated that the excitatory inputs of SL neurons are the most selective, in contrast to the excitatory inputs of SS and SA neurons and the inhibitory inputs of all the three types of neurons (Fig. 6B; excitatory inputs (E); inhibitory inputs (I); onetail two-sample $t$ test: MT $+10 \mathrm{~dB}$, E-SL vs E-SS: $t_{(23)}=1.83, p=$ 0.0399 , E-SL vs E-SA: $t_{(21)}=2.24, p=0.0182$, E-SL vs I-SS: $t_{(23)}=$ $3.00, p=0.0032$, E-SL vs I-SA: $t_{(21)}=2.31, p=0.0155$, E-SL vs I-SL: $t_{(24)}=2.41, p=0.0119 ; \mathrm{MT}+20 \mathrm{~dB}$, E-SL vs E-SS: $t_{(23)}=$ $2.95, p=0.0036$, E-SL vs E-SA: $t_{(21)}=2.98, p=0.0035, \mathrm{E}-\mathrm{SL}$ vs I-SS: $t_{(23)}=3.50, p=0.0010$, E-SL vs I-SA: $t_{(21)}=3.40, p=$ 0.0013 , E-SL vs I-SL: $t_{(24)}=2.96, p=0.0034 ; \mathrm{MT}+30 \mathrm{~dB}$, E-SL vs E-SS: $t_{(23)}=2.31, p=0.0150$, E-SL vs E-SA: $t_{(21)}=2.41, p=$ 0.0127 , E-SL vs I-SS: $t_{(22)}=4.46, p=0.0001$, E-SL vs I-SA: $t_{(21)}=$ $3.31, p=0.0017$, E-SL vs I-SL: $t_{(24)}=2.64, p=0.0071 ; \mathrm{MT}+40$ $\mathrm{dB}, \mathrm{E}-\mathrm{SL}$ vs E-SS: $t_{(21)}=3.99, p=0.0003$, E-SL vs E-SA: $t_{(19)}=$ $3.11, p=0.0028$, E-SL vs I-SS: $t_{(21)}=4.40, p=0.0001, \mathrm{E}-\mathrm{SL}$ vs I-SA: $t_{(20)}=3.57, p=0.0010$, E-SL vs I-SL: $t_{(20)}=4.600, p=$ $0.0004)$. SS neurons had well balanced excitatory and inhibitory TRFs and frequency tuning curves, as both $\mathrm{E} / \mathrm{I}$ ratio and $\mathrm{E} / \mathrm{I}$ ratio $_{50}$ were close to one (Fig. 6C). SA neurons had matched excitatory and inhibitory TRFs but unmatched frequency tunings, with $\mathrm{E} / \mathrm{I}$ ratios close to one and larger $\mathrm{E} / \mathrm{I}$ ratio $_{50}$ (Fig. $6 \mathrm{C}$; $\mathrm{E} / \mathrm{I}$ ratio vs $\mathrm{E} / \mathrm{I}$ ratio $_{50}$, one-tail paired $t$ test, $\left.t_{(9)}=-7.37, p<0.0001\right)$. SL neurons had imbalanced excitation and inhibition with narrower excitatory TRFs and frequency tuning, as indicated by small E/I ratios and $\mathrm{E} / \mathrm{I} \mathrm{ratio}_{50}$ (Fig. $6 \mathrm{C}$ ).

To examine the temporal relationship between excitatory and inhibitory synaptic inputs, their onset latency at CF ("At CF", determined by spike TRFs), one octave lower than CF ("Below CF"), and one octave higher than CF ("Above CF") were measured. If the left or right boundaries of TRFs were less than one octave from the CF (especially for SL neurons), the responses at the boundaries were used for analysis. The onset latency of excitatory and inhibitory inputs below $\mathrm{CF}$ and at $\mathrm{CF}$ was not significantly different among the three types of neurons (Fig. 6D, E; excitatory, one-way ANOVA, Below CF: $F_{(2,32)}=1.83, p=$ 0.1768; At CF: $F_{(2,32)}=1.47, p=0.2440$; inhibitory, Below CF: $F_{(2,32)}=1.45, p=0.2489 ;$ At CF: $\left.F_{(2,32)}=2.15, p=0.1329\right)$, but that above CF was significantly different (excitatory, one-way ANOVA: $F_{(2,32)}=11.29, p=0.0002$; inhibitory, one-way ANOVA: $\left.F_{(2,32)}=4.79, p=0.0151\right)$. As the relative timing between inhibition and excitation plays important roles on sensory processing (Wehr and Zador, 2003; Wu et al., 2006), we further examined whether it was different within the synaptic TRFs of sensitive and selective neurons (Fig. $6 F$ ). Our data showed that SS neurons had a lagged inhibition across their synaptic TRFs (onetail paired $t$ test, excitatory vs inhibitory, Below CF: $t_{(11)}=-4.33$, $p=0.0006$; At CF: $t_{(11)}=-4.18, p=0.0008$; Above CF: $t_{(11)}=$ $-4.54, p=0.0004)$; SA neurons had a lagged inhibition below $\mathrm{CF}$, but concurrent excitation and inhibition above CF (one-tail paired $t$ test, excitatory vs inhibitory, Below CF: $t_{(9)}=-7.65, p<$ 0.0001; At CF: $t_{(9)}=-2.50, p=0.0169$; Above CF: $t_{(9)}=-0.57$, $p=0.2915$ ); whereas SL neurons have concurrent excitatory and inhibitory inputs across the synaptic TRFs (one-tail paired $t$ test, excitatory vs inhibitory, Below CF: $t_{(12)}=0.63, p=0.2688$; At CF: $t_{(12)}=-0.65, p=0.2629$; Above CF: $t_{(12)}=0.35, p=$ 0.3650 ). For SA neurons, the asymmetry of spike TRFs was enhanced in contrast to that of excitatory TRFs (Fig. 6G). The CFs measured from excitatory and inhibitory synaptic TRFs were well correlated for SS and SA neurons, but not for SL neurons (Fig. 6H). Our data imply that the spectrotemporal configurations between excitation and inhibition could be highly diverse to further differentiate the effects of inhibition within the synaptic TRFs.

\section{Spectrotemporal interaction between excitation and inhibition shapes spike TRFs}

To examine how the synaptic strength and relative timing of inhibitory inputs contribute to the segregation of CNIC neurons' frequency selectivity, we adopted a single-compartment integrateand-fire neuron model to integrate excitatory and inhibitory inputs within synaptic TRFs, and to simulate suprathreshold membrane potential responses (Wehr and Zador, 2003; Zhou et al., 2010; Kuo and $\mathrm{Wu}, 2012$ ). By comparing the simulated spike TRFs with the actual spike TRFs, this strategy offers a unique opportunity to validate the potential synaptic mechanisms and detect potential measurement errors in in vivo voltage-clamp recordings. For sensitive neurons, the simulated spike TRFs were largely overlapped with their actual spike TRFs (Fig. $7 A, B$ ). It is worth noting that the spike TRFs of SS neurons also largely overlapped with their excitatory TRFs (Fig. 7A). For SA neurons, the low-frequency boundary of spike TRFs was similarly shifted toward CF compared with that of excitatory synaptic TRFs at all sound intensities, but their highfrequency boundary of spike TRFs was shifted to a greater extent at higher intensities (Fig. 7B). It created a steeper high-frequency slope that exacerbated the asymmetry of spike TRFs for SA neurons (Fig. $6 G$ ). The simulated spike TRFs of SL neurons were smaller than their excitatory synaptic TRFs, but larger than their actual spike TRFs, implying that both inhibition and other mechanisms may together enhance their selectivity (Fig. 7C).

Intrinsic properties, such as spike generation mechanisms, could further shape the spike responses (Baker, 2001; Priebe and Ferster, 2008; Gittelman et al., 2009). As the simulated spike TRF of the SL neuron was overrepresented in Figure $6 F$, we adjusted spike thresholds from 5 to $25 \mathrm{mV}$ to examine whether firing thresholds could affect spike TRFs. The size of spike TRFs only varied by $5-10 \%$ for SS and SA neurons, but the linearity between frequency bandwidth and sound intensity was largely reduced for SL neurons when the firing threshold was increased to $20 \mathrm{mV}$, showing a further narrowed frequency range (Fig. $7 D-F$ ). These data suggest that mechanisms such as thresholding may further improve selectivity for selective neurons, in addition to the inhibitory synaptic mechanism.

\section{In vivo assessment of the effects of space-clamping errors}

A long-lasting concern of synaptic currents measured by voltageclamp recordings stems from the space-clamping errors -the failure to clamp neuron's membrane potential at distal dendrites and dendritic spines (Spruston et al., 1993; Williams and Mitchell, 2008). Although such errors could be estimated by computational models with data from in vitro slice recordings, it is not feasible with in vivo recordings (Beaulieu-Laroche and Harnett, 2018). In our study, $I-V$ curves of the recorded neurons suggest that cells were reasonably clamped, and the synaptic currents were not strongly affected by the nonlinearities of the neurons (Fig. 8A,B).

To further examine the potential effects of space-clamping errors on synaptic currents in vivo, we simulated membrane po- 

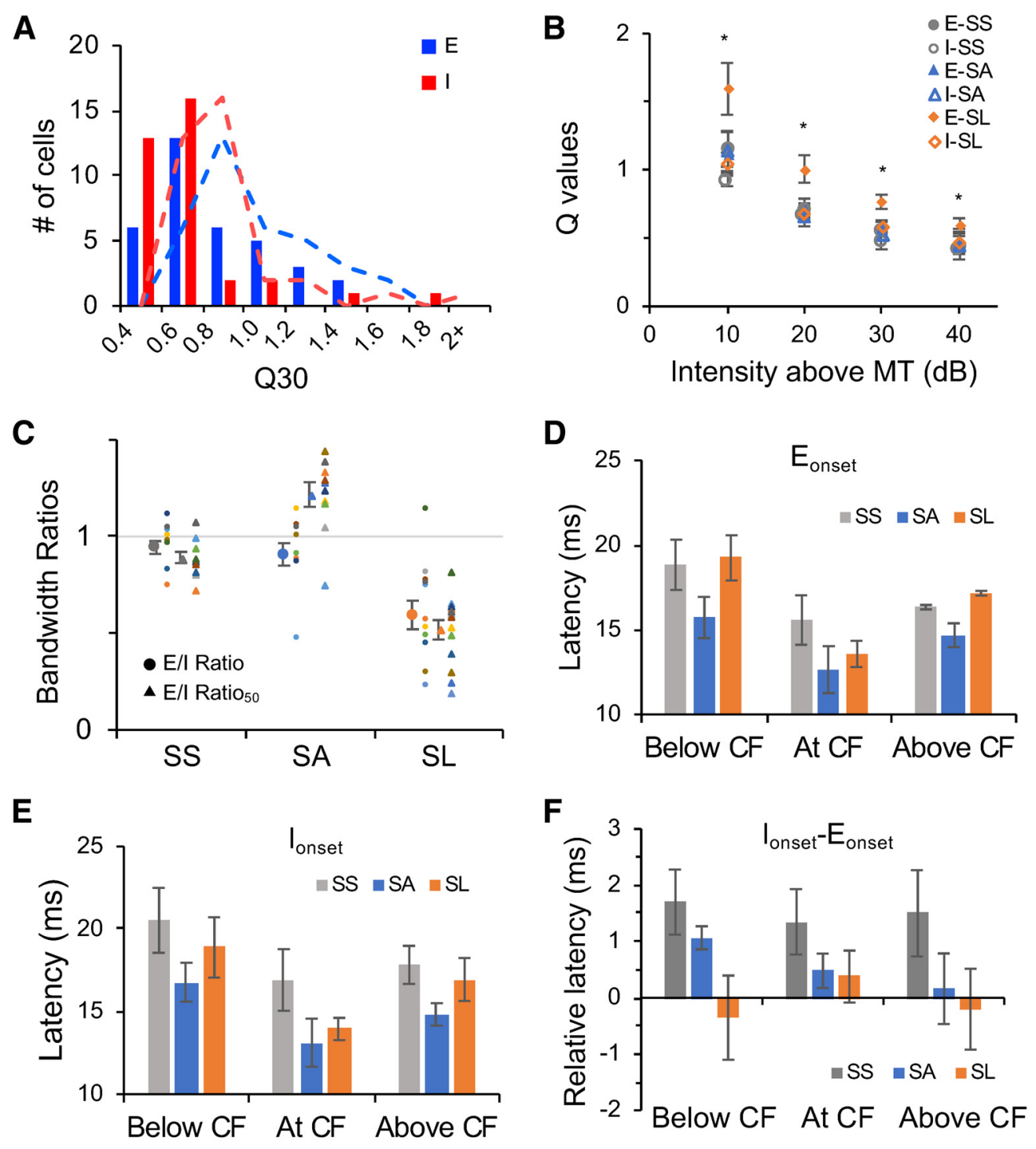

G

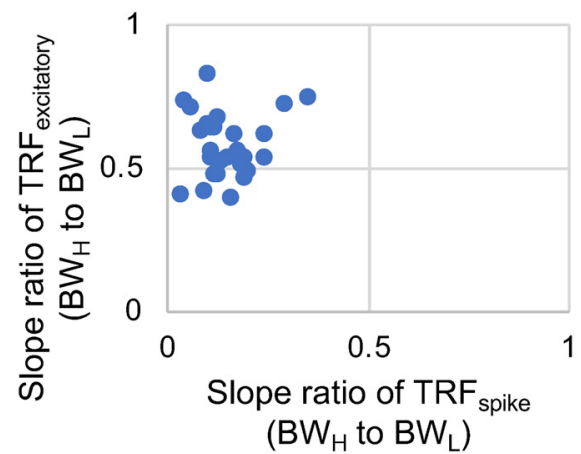

H

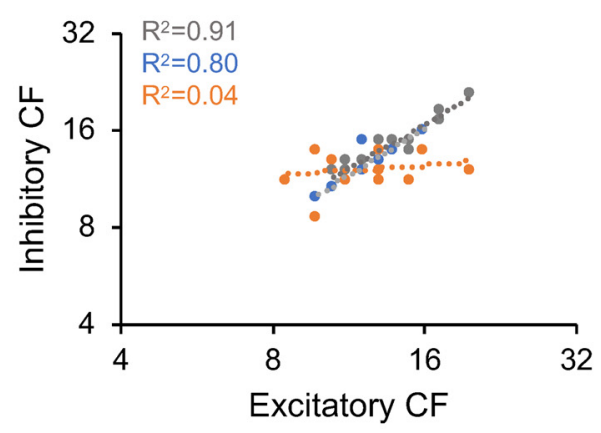

Figure 6. Frequency selectivity, and relative timing of excitatory and inhibitory inputs for SS, SA and SL neurons. A, Distribution of Q30 values of excitatory (blue) and inhibitory (red) TRFs. Dashed trend line indicates moving average of two nearby bins. $\boldsymbol{B}, Q$ values of excitatory and inhibitory TRFs at different sound intensities for $S S(n=12)$, sensitive-asymmetrical $S A(n=10)$ and selective $\mathrm{SL}(n=13)$ neurons. Asterisks indicate significant larger $Q$ values of excitatory inputs of $S \mathrm{~L}$ neurons. $\mathrm{C}, \mathrm{E} / \mathrm{l}$ ratios and $\mathrm{E} / \mathrm{I}$ ratio ${ }_{50} \mathrm{for} S \mathrm{SS}, \mathrm{SA}$, and $\mathrm{SL}$ neurons. Circle indicates the mean of $\mathrm{E} / \mathrm{I}$ ratio, triangle indicates the mean of E// ratio $0_{50}$. Error bar indicates SEM. SS, Gray; SA, blue; SL: orange. Colored dots indicate individual neurons. D, Onset latency of excitatory inputs below CF, at CF, and above CF for SS $(n=12), S A(n=10)$, and SL $(n=13)$ neurons. Below CF and Above CF were selected from responses evoked by tones at 1 octave below or above CFs. If the distance between frequency boundaries and CFs was $<1$ octave, then the responses at the boundaries were selected. Error bar indicates SEM. E, Onset latency of inhibitory inputs below CF, at CF, and above CF for SS, SA, and SL neurons. Error bar indicates SEM. $\boldsymbol{F}$, Relative onset latency between excitatory and inhibitory inputs (inhibitory - excitatory) below CF, at CF, and above CF for SS, SA, and SL neurons. Error bar indicates SEM. G, The relationship between the asymmetry of spike TRFs and that of excitatory synaptic TRFs for SA neurons (BW to $_{\mathrm{H}} \mathrm{BW}_{\mathrm{L}}$; see Materials and Methods; Fig. 1C), indicating strengthened asymmetry of spike TRFs. $\boldsymbol{H}$, The relationship between the CFs of excitatory TRFs and those of inhibitory TRFs. SS, Gray; SA, blue, SL, orange. 
A

SS neuron

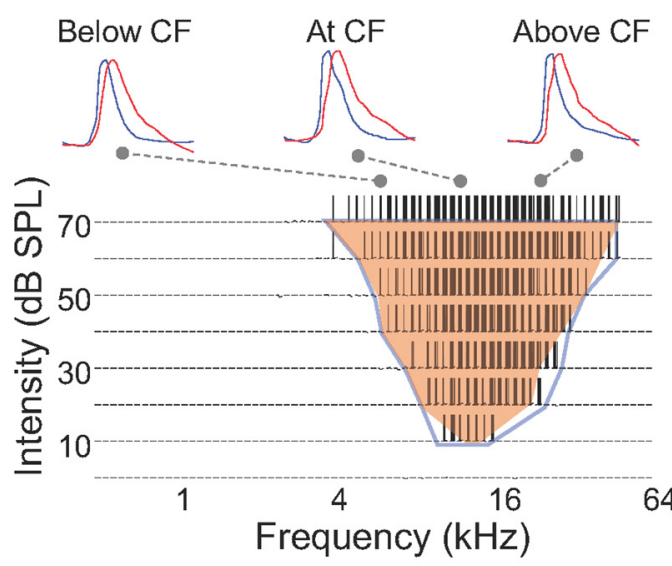

B
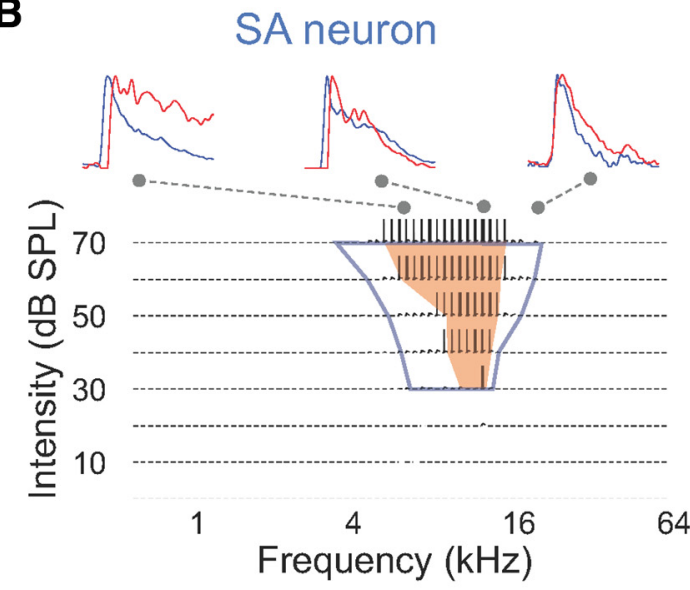

C

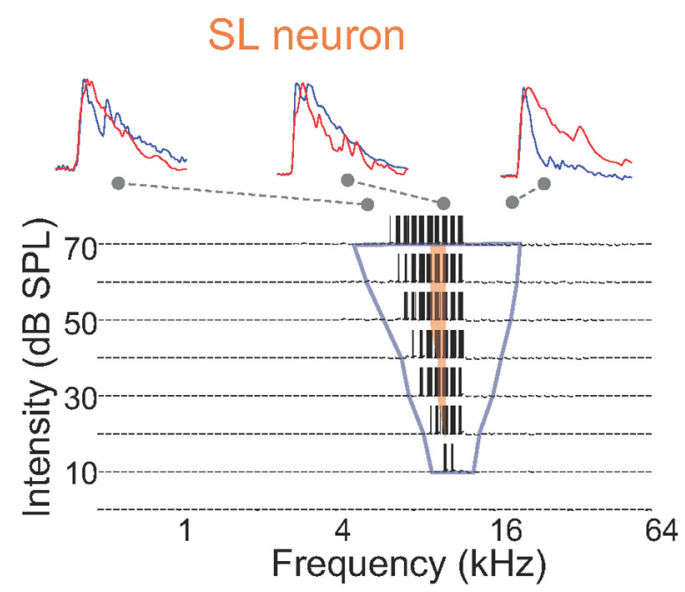

D

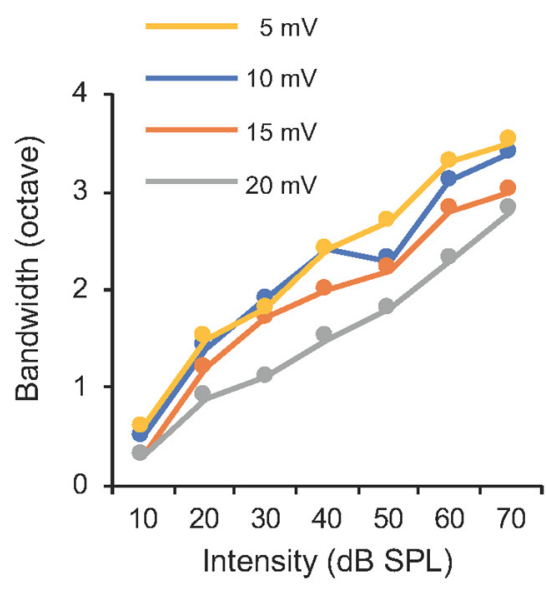

E

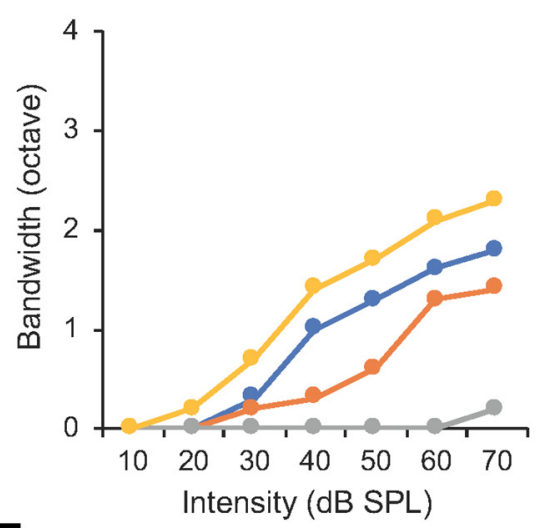

F

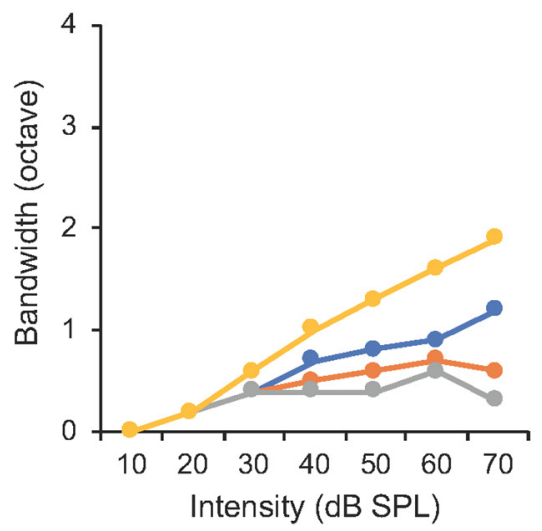

Figure 7. The effects of inhibition and thresholding to frequency selectivity. $A$, Top traces, Individual excitatory (blue) and inhibitory (red) inputs below CF, at CF, and above CF for the SS neuron in Figures 3 and 5 . Bottom, the TRF of simulated spike responses by integrating excitatory and inhibitory inputs in Figure $5 A$ and $B$. Connecting dashed lines indicate the location of individual traces in the TRFs. Blue contour delineates the boundary of excitatory synaptic TRF, orange shaded area indicates the actual spike TRF from cell-attached recordings. $\boldsymbol{B}$, Same as $\boldsymbol{A}$, but for the case in Figure 5, C and D. C, Same as $\boldsymbol{A}$, but for the case in Figure 5, E and F. D, The effects of different spike thresholds on the frequency bandwidths of spike TRFs for the SS neuron in $\boldsymbol{A}$. $\boldsymbol{E}$, Same as $\boldsymbol{D}$, but for the SA neuron in $\boldsymbol{B}$. $\boldsymbol{F}$, Same as $\boldsymbol{D}$, but for the SL neuron in $\boldsymbol{C}$.

tentials when attenuating excitatory and inhibitory inputs or adjusting their temporal relationship (Fig. $8 C-E$ ). The peak of simulated membrane potential responses was quickly diminished when excitatory inputs were attenuated $>50 \%$ and other parameters (e.g., inhibitory inputs and relative latency) were held constant (Fig. 8C). But it gradually increased when inhibitory inputs were further attenuated or delayed (Fig. $8 D, E$ ). This result indicates that the integrate-and-fire model is sensitive to the attenuation of synaptic inputs and the distorted relative timing of excitatory and inhibitory inputs. An underrepresentation of spike responses and smaller spike TRFs would have occurred if space-clamping errors had severely distorted synaptic currents in 
A
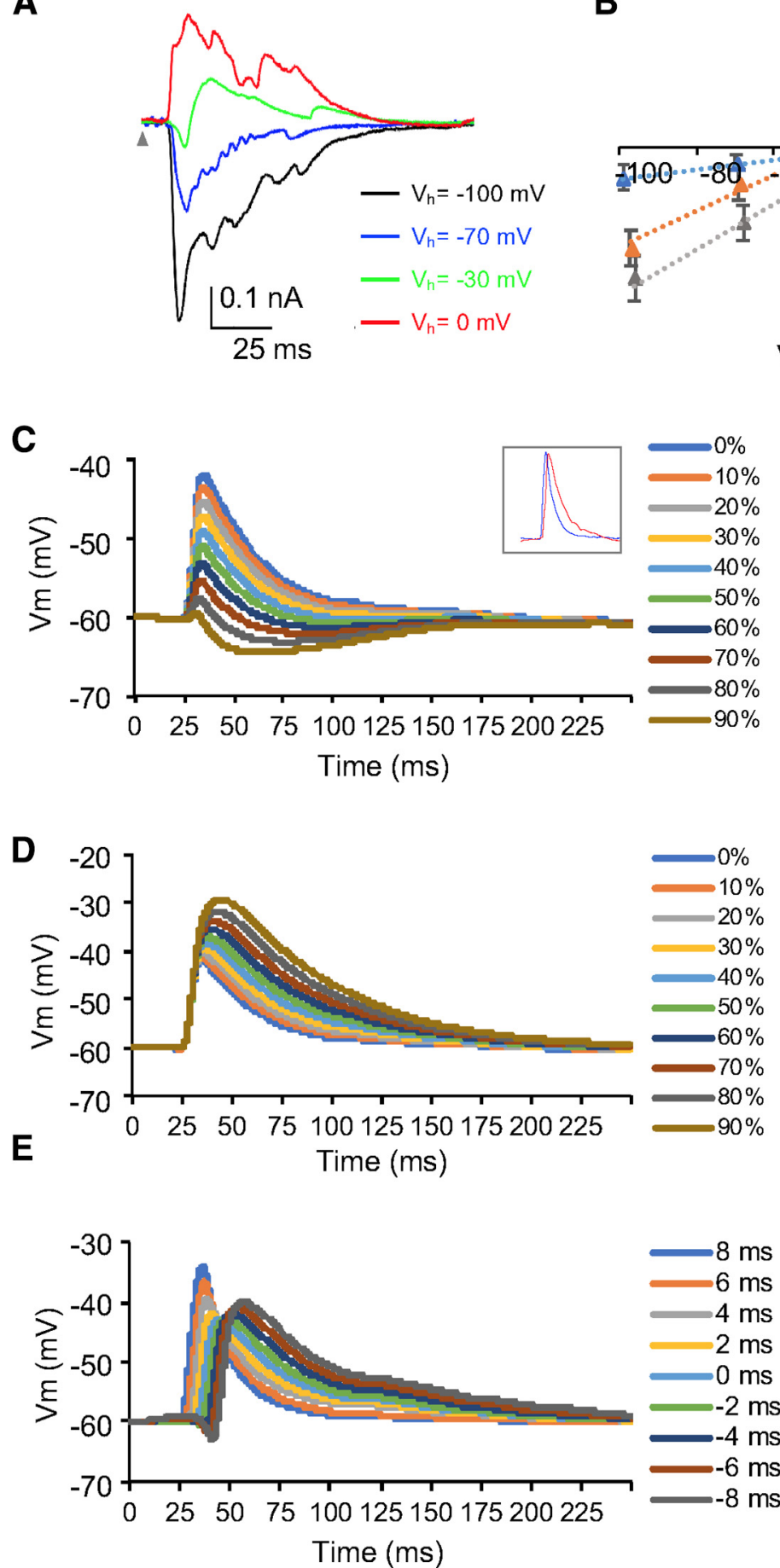
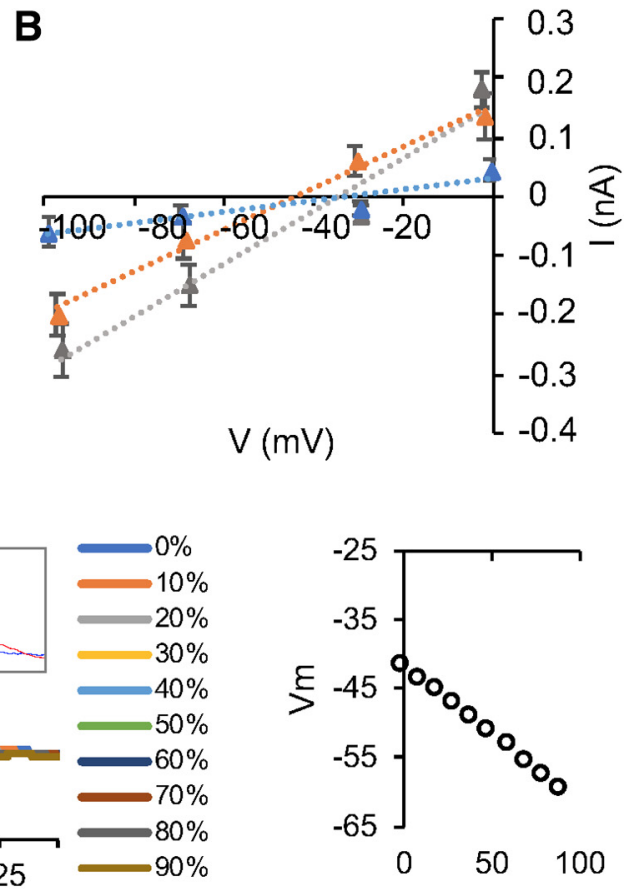

$\%$ E Attenuation
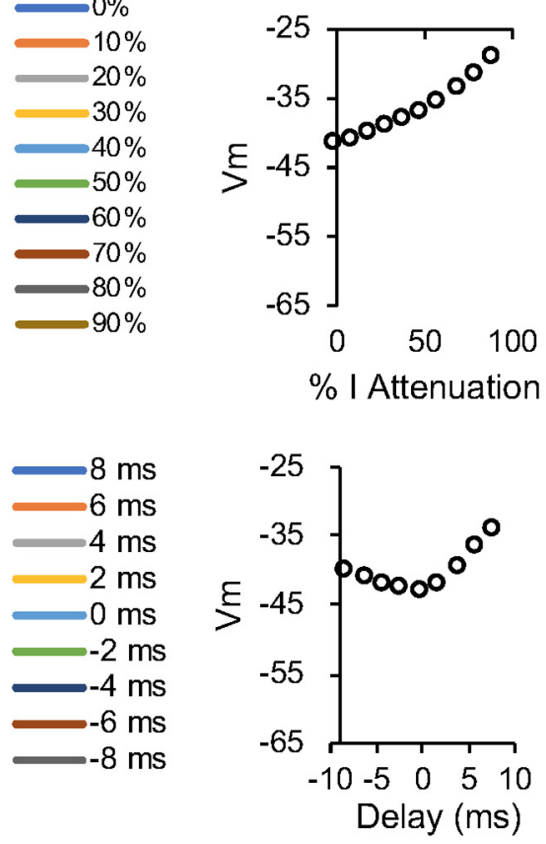

Figure 8. The effects of synaptic currents attenuated by space-clamping errors on membrane potential responses. $A$, Synaptic currents evoked by CF tones ( $16 \mathrm{kHz}$ ) at $60 \mathrm{~dB}$ SPL when the membrane potential of the example SS neuron was clamped at different holding voltages. Gray arrowhead indicates tone onset. $\boldsymbol{B}, I-V$ relationships for the three neurons in Figure $5 A-F$. I-V curves for synaptic currents averaged within a time window between 13 and 15 ms after the stimulus onset. M \pm SD. SS, Gray; SA, blue; SL: orange. C, Simulated membrane potential responses when excitatory inputs were attenuated by $10-90 \%$. Data point at $0 \%$ indicates simulation from non-attenuated synaptic inputs. Inset, Non-attenuated EPSC (blue) and IPSC (red) used in simulation. D, Simulated membrane potential responses when inhibitory inputs were attenuated by $10-90 \%$. Data point at $0 \%$ indicates simulation from non-attenuated synaptic inputs. $\boldsymbol{E}$, Simulated membrane potential responses when the relative latency between excitatory and inhibitory inputs were adjusted between -8 and 8 ms (inhibitory - excitatory, negativity indicates leading inhibition).

voltage-clamp recordings. However, this was not observed from our analysis (Fig. 7). Except for the false positivity of selective neurons from overrepresented spike responses, the false-positive and false-negative percentages of simulated spike responses were $<10 \%$ for sensitive neurons. Therefore, this strategy of combin- ing in vivo cell-attached recording, voltage-clamp recording, and computational modeling can validate synaptic mechanisms in the CNIC to a great extent.

Q30 of simulated spike TRFs recovered a differentiated frequency selectivity from undifferentiated synaptic inputs when 
A

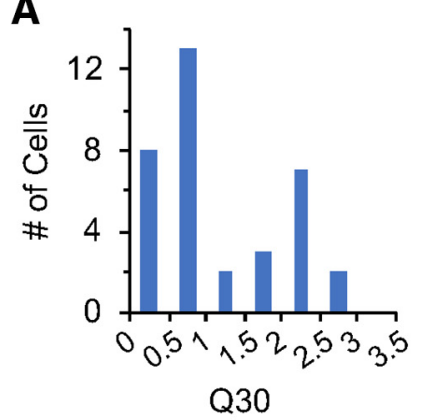

D

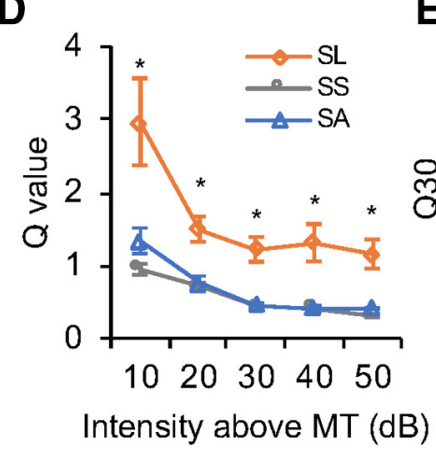

B

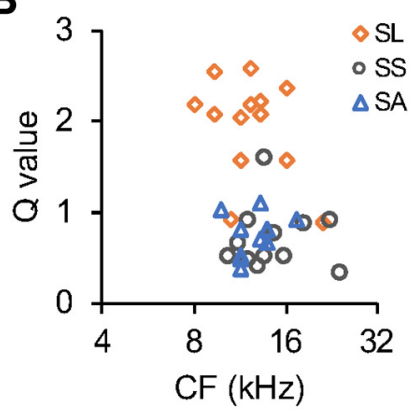

$\mathbf{E}$

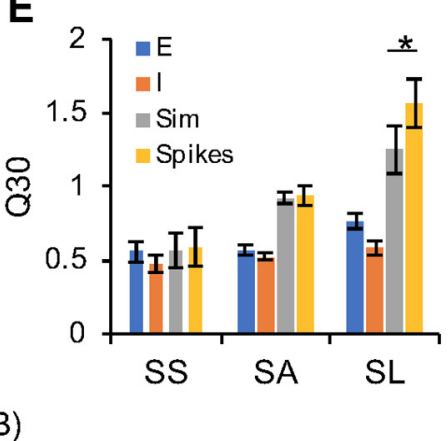

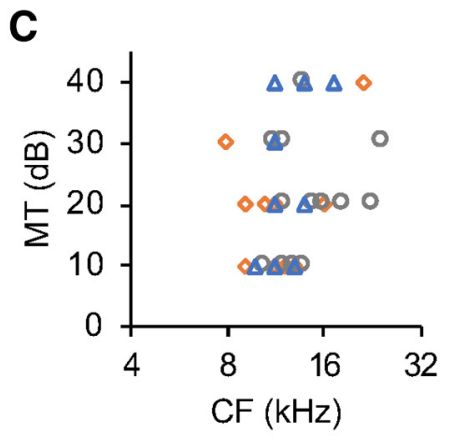

F

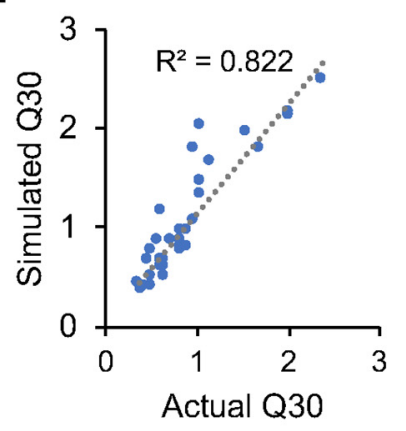

Figure 9. Frequency selectivity of simulated and recorded spike responses. $\boldsymbol{A}$, Distribution of Q30 values of simulated spike TRFs. $\boldsymbol{B}$, Scatter plot of Q30 values of simulated spike TRFs versus CFs. C, Scatter plot of MTs of simulated spike TRFs versus CFs. D, Q values of simulated spike TRFs at different sound intensities above MT for sensitive and selective neurons. Asterisk indicates significance of SL versus SS and SL versus SA. E, Q30 values of excitatory inputs, inhibitory inputs, simulated spike (Sim), and recorded actual spike (Spikes) for the three types of neurons. Asterisks indicate significance. $\boldsymbol{F}, \mathbf{Q 3 0}$ values of simulated spike TRFs versus that of recorded actual spike TRFs.

the firing threshold was set to $10 \mathrm{mV}$ for all the cells (Figs. $4 A, 9 A$ ). This suggests that the inhibitory mechanism may have already been sufficient to segregate sensitive and selective neurons even without a further enhancement by the cell's intrinsic properties. Simulated spike TRFs also showed that SL neurons in general had larger $Q$ values than SS and SA neurons, indicating greater selectivity (Fig. $9 B, D$; one-tail two-sample $t$ test, MT $+10 \mathrm{~dB}$, SL vs SS: $t_{(23)}=3.21, p=0.0019$, SL vs SA: $t_{(21)}=2.53, p=0.0098$, SA vs SS: $t_{(20)}=1.86, p=0.0389 ; \mathrm{MT}+20 \mathrm{~dB}$, SL vs SS: $t_{(23)}=4.20, p=$ 0.0002 , SL vs SA: $t_{(21)}=2.65, p=0.0075$, SA vs SS: $t_{(20)}=1.68$, $p=0.0541 ; \mathrm{MT}+30 \mathrm{~dB}$, SL vs SS: $t_{(23)}=5.32, p<0.0001, \mathrm{SL}$ vs SA: $t_{(21)}=4.90, p<0.0001$, SA vs SS: $t_{(20)}=0.51, p=0.3094$; $\mathrm{MT}+40 \mathrm{~dB}$, SL vs SS: $t_{(22)}=3.44, p=0.0012$, SL vs SA: $t_{(19)}=$ $2.68, p=0.0073$, SA vs SS: $t_{(19)}=1.60, p=0.0628 ; \mathrm{MT}+50 \mathrm{~dB}$, SL vs SS: $t_{(18)}=3.37, p=0.0017$, SL vs SA: $t_{(17)}=2.81, p=$ 0.0061 , SA vs SS: $\left.t_{(15)}=1.56, p=0.0698\right)$, although their MTs were not significantly different (Fig. $9 C$; one-way ANOVA: $F_{(2,32)}$ $=0.4402, p=0.6477)$. This is similar to the analysis of actual spike TRFs in Figure 4. The simulated TRFs of sensitive neurons had similar frequency ranges to their actual TRFs (two-tail paired $t$ test, SS: $t_{(12)}=0.84, p=0.4195$; SA: $\left.t_{(9)}=0.39, p=0.7020\right)$, whereas selective neurons typically showed broader simulated spike TRFs and smaller Q values than their actual spike TRFs (Fig. $9 E$; two-tail paired $t$ test, SL: $\left.t_{(12)}=3.76, p=0.0027\right)$. Overall, frequency bandwidths of simulated TRFs and actual TRFs were well correlated, suggesting minimal effects of space-clamping errors in our recordings (Fig. $9 F$ ).

\section{Discussion}

A capacity of neural ensembles to optimize their sensitivity and selectivity

Sensitivity and selectivity have to complement each other in sensory systems. Toward this end, a parallel processing may be im- plemented to simultaneously detect and distinguish a wide range of behaviorally important stimuli by neural ensembles with broad receptive fields and sharp tuning curves (Hubel and Wiesel, 1962; Møller, 2003; Priebe and Ferster, 2008; Kato et al., 2017). In the mouse CNIC, neural populations with different CFs had low MTs (between 10 and $20 \mathrm{~dB}$ SPL) and broad frequency ranges (as broad as 4 octaves, $\sim$ one-third of mouse's hearing frequency range; Fig. $2 F, G$ ). It cannot be attributed to a nonspecific sampling of neurons from multiple iso-frequency laminae in the CNIC, because our configuration of multiunit recordings typically obtained spike responses within a diameter of 150-200 $\mu \mathrm{m}$ from the electrode tips (Saleem et al., 2017). This distance corresponds to a single iso-frequency lamina and a CF's change of $<1$ octave in the CNIC of mice (Willott and Urban, 1978; Stiebler and Ehret, 1985; Malmierca et al., 2008). It is also obvious that neural populations' broad TRFs did not compromise their frequency selectivity, as neural populations with CFs between 8 and $22 \mathrm{kHz}$ had similar responding frequency range but differential frequency tunings and sharper selectivity (Fig. $2 D, F$ ). Interestingly, behavioral audiograms of mice showed peak sensitivity between 8 and 24 kHz (Fig. 1G; Ehret, 1974; Zheng et al., 1999; Prosen et al., 2003; Radziwon et al., 2009). This implies that neural populations may strategically allow an easy detection and an efficient identification of incoming stimuli in this behavioral frequency range by collecting individual neurons' diverse sensitivity and selectivity.

\section{A divergence of individual neurons' frequency selectivity}

Neurons' spike TRFs are highly diverse in the CNIC, which may represent general principles for optimizing auditory selectivity in mice (Egorova et al., 2001; Hage and Ehret, 2003), rats (Malmierca et al., 2008), cats (Ehret and Merzenich, 1988; Ramachandran et al., 1999), guinea pigs (LeBeau et al., 2001), and bats 
A

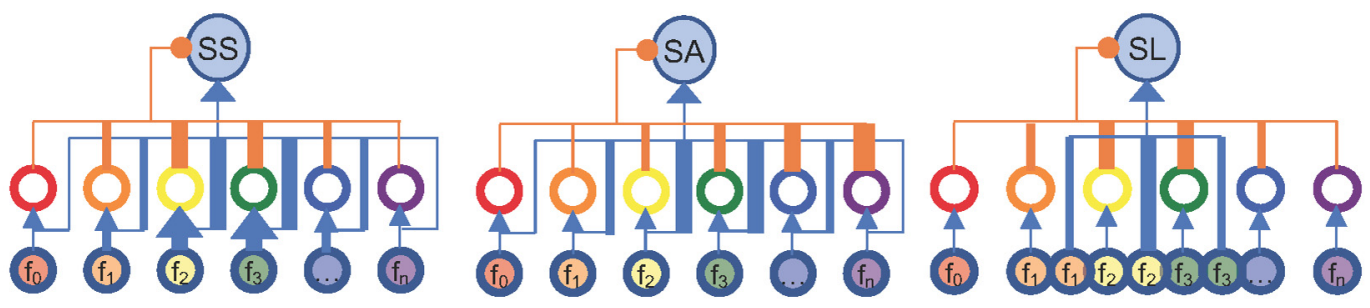

B

Tuning curves of synaptic inputs
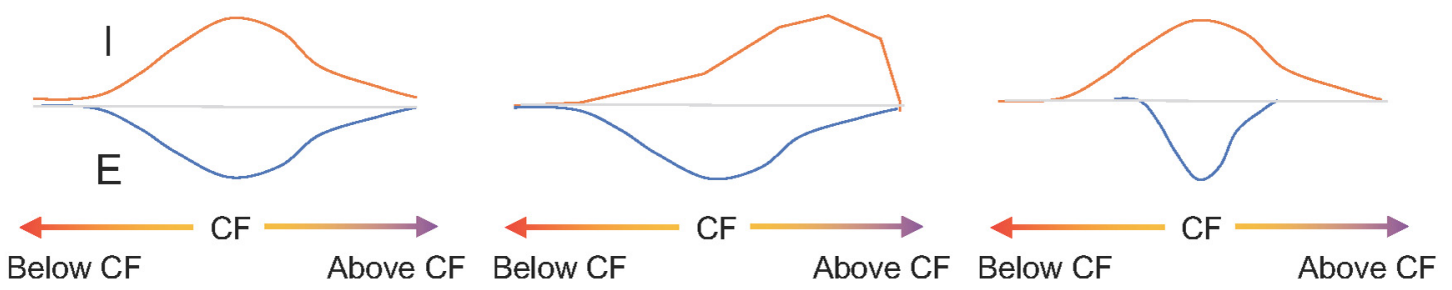

Figure 10. Schematic drawing of potential neural circuits for sensitive and selective neurons. $A$, Neural circuits for $S S, S A$, and $S L$ neurons. $f_{0}, f_{1}, f_{2} \ldots$ to $f_{n}$ indicate different frequency channels, thickness represent synaptic strengths with excitatory projections in blue and inhibitory innervations in orange. $\boldsymbol{B}$, Excitatory (blue) and inhibitory (orange) frequency tuning curves for SS, SA, and SL neurons.

(Portfors and Wenstrup, 2002). We adopted simultaneous multiunit and cell-attached recordings to guarantee a faithful identification of single-cell activities from a population background in vivo (Kuo and $\mathrm{Wu}, 2012$ ). Although anesthesia may affect the abundance of each different cell type or their spontaneous activities, major types of spike TRFs have long been identified in the CNIC of animals in an awake condition or under different anesthesia (Stiebler and Ehret, 1985; Ramachandran et al., 1999; Egorova et al., 2001; LeBeau et al., 2001; Portfors et al., 2011). SS, SA, and SL neurons may respectively correspond to type III, type I, and type II neurons in previous studies, whereas type IV neurons (with complex or enclosed spike TRFs) were not encountered in our sample, possibly because of their sparseness for more specialized functions in mice (Egorova et al., 2001; Lu and Jen, 2001). Interestingly, most of SL neurons' CFs were between 8 and 22 $\mathrm{kHz}$ (Fig. $4 B$ ). Since the aforementioned neural populations in the CNIC and behavioral audiogram showed the lowest MTs in this hearing range of mice, SL neurons may represent a functional subpopulation to maintain strong selectivity in neural ensembles with great sensitivity (Ehret, 1974; Zheng et al., 1999; Radziwon et al., 2009).

In addition to differentiated frequency representation, SS, SA and SL neurons may be further investigated for other specialized functions such as duration tuning (Aubie et al., 2012; Alluri et al., 2016), tone interval selectivity (Edwards et al., 2002), or complex sound analysis (Portfors and Wenstrup, 2002; Portfors et al., 2011; Mayko et al., 2012). Because SA and SL neurons showed imbalanced excitatory and inhibitory synaptic TRFs, they may also be selective to the directions of frequency-modulated sweeps (Gittelman et al., 2009; Kuo and Wu, 2012). In the future, these specialized functional and anatomical subpopulations may be mapped within CF-defined neural ensembles. A recent study showed that GABAergic and glutamatergic neurons in the CNIC of mice cannot be distinguished by electrophysiological criteria, such as spike waveforms, TRFs, or MTs (Ono et al., 2017). Thus, it is likely that neurons in our study were recorded from a collection of different neurotransmitter and morphological subtypes. As the main focus of our study was to investigate diversified frequency representation of CNIC neurons, the uncovered syn- aptic mechanisms may reflect a general principle despite neurons' neurotransmitter expression or morphology.

\section{A synaptic mechanism of separating sensitive and selective neurons}

Our strategy to combine in vivo cell-attached and voltage-clamp recordings allowed us to directly assess the synaptic mechanisms by associating spike and synaptic responses from the same neurons. The spectrotemporal interaction between excitatory and inhibitory inputs has been proposed as a major synaptic mechanism for sensory processing and neurons' functional heterogeneity (Isaacson and Scanziani, 2011; Kuo and Wu, 2012; Tao et al., 2017). Our results revealed that at least three spectral configurations of excitatory and inhibitory inputs coexist for nearby CNIC neurons: (1) matched excitatory and inhibitory synaptic TRFs with matched excitatory and inhibitory frequency tuning; (2) matched excitatory and inhibitory synaptic TRFs with sharper inhibitory tuning; and (3) mismatched excitatory and inhibitory synaptic TRFs with broader inhibitory tuning. As GABAergic neurons with different frequency bandwidths have been reported recently, they may contribute to our observed diverse inhibitory configurations (Ono et al., 2017).

In contrast to the constant latency between excitatory and inhibitory inputs in feedforward inhibitory circuit models (Wehr and Zador, 2003; Zhang et al., 2003), the temporal configuration of excitatory and inhibitory inputs was highly variable within the TRFs of CNIC neurons (Fig. 6F). Inhibitory inputs either followed or preceded excitatory inputs, which could globally control spike timing for SS and SA neurons or selectively scale down the responses of SA and SL neurons to certain frequencies (Fig. 7; Zhou et al., 2015). Thus, it is reasonable to assume that heterogeneous inhibitory circuits coexist locally to differentiate neurons' functionality such as frequency selectivity in the CNIC (Fig. 10).

However, other mechanisms cannot be completely ruled out, as neurons' intrinsic properties could also determine their frequency selectivity by scaling down excitability or thresholding membrane responses (Kuo and $\mathrm{Wu}, 2012$; Ono and Oliver, 2014b). Particularly for selective neurons in our study, computa- 
tional integration of excitation and inhibition did not narrow the simulated spike TRFs to the size of actual TRFs (Fig. 7C), although they were still well correlated (Fig. 9E,F). Moreover, the firing patterns could not be recovered from our computational models, whereas both regular and fast spiking neurons were observed in the CNIC (Wagner, 1994; Peruzzi et al., 2000).

\section{An estimation of synaptic contribution spared from space-clamping errors}

As the interpretation of synaptic mechanisms is heavily dependent on the analyses of synaptic inputs, the only applicable technique to acquire EPSCs and IPSCs has been voltage-clamp recording so far. It is unfortunately subject to the scrutiny of measurement errors (Spruston et al., 1993; Williams and Mitchell, 2008). Previous evaluations of space-clamping errors were based on the theoretical and modeling analyses of large pyramidal neurons in the layer 5 of the neocortex from brain slice preparations, which is not feasible for in vivo recordings of neurons with diverse morphology (Beaulieu-Laroche and Harnett, 2018).

To inspect the potential effects of space-clamping errors on the validation of synaptic mechanisms in vivo, we propose a practical strategy that computationally models spike responses from recorded synaptic inputs and compares them with the actual spike recordings. If the synaptic inputs had been severely distorted in in vivo voltage-clamp recordings, the simulated and actual spike TRFs would inevitably have shown great discrepancies. In our data, however, the simulated spike TRFs were well correlated with the actual recorded spike TRFs. It suggests that the spectrotemporal interactions between excitatory and inhibitory inputs are the basis for differentiating the frequency selectivity of CNIC neurons. The low impact from space-clamping errors in our recordings may be because of the smaller spine resistance, distribution of spine locations, shorter dendritic arbors, smaller cell bodies in the mouse CNIC, and relatively intact dendritic or axonal processes of recorded neurons unlike in in vitro recordings (Peruzzi et al., 2000; Ono et al., 2005, 2017; Ito et al., 2009).

In summary, our strategy of combining in vivo multiunit, cellattached, and voltage-clamp recordings allowed us to bridge the analysis at different levels such as neural populations, single cells, and synapses with sufficient spatiotemporal resolutions. Thus, the synaptic mechanisms can be examined with a greater extent of validity. Our results pinpoint that neural circuits could be configured to create differential relative strength and timing of excitatory and inhibitory inputs within synaptic TRFs, and to specify various selectivity of nearby individual neurons. As CNIC neurons start to be sensitive or selective to frequency-modulated and vocal signals, our result also shed light on the neural circuitry mechanisms for complex sound processing (Kuo and $\mathrm{Wu}, 2012$; Woolley and Portfors, 2013).

\section{References}

Aitkin L, Tran L, Syka J (1994) The responses of neurons in subdivisions of the inferior colliculus of cats to tonal, noise and vocal stimuli. Exp Brain Res 98:53-64.

Alkhatib A, Biebel UW, Smolders JW (2006) Inhibitory and excitatory response areas of neurons in the central nucleus of the inferior colliculus in unanesthetized chinchillas. Exp Brain Res 174:124-143.

Alluri RK, Rose GJ, Hanson JL, Leary CJ, Vasquez-Opazo GA, Graham JA, Wilkerson J (2016) Phasic, suprathreshold excitation and sustained inhibition underlie neuronal selectivity for short-duration sounds. Proc Natl Acad Sci U S A 113:E1927-E1935.

Anderson JS, Carandini M, Ferster D (2000) Orientation tuning of input conductance, excitation, and inhibition in cat primary visual cortex. J Neurophysiol 84:909-926.
Aubie B, Sayegh R, Faure PA (2012) Duration tuning across vertebrates. J Neurosci 32:6373-6390.

Baker CL Jr (2001) Linear filtering and nonlinear interactions in directionselective visual cortex neurons: a noise correlation analysis. Vis Neurosci 18:465-485.

Beaulieu-Laroche L, Harnett MT (2018) Dendritic spines prevent synaptic voltage clamp. Neuron 97:75-82.e3.

Beyerl BD (1978) Afferent projections to the central nucleus of the inferior colliculus in the rat. Brain Res 145:209-223.

Caspary DM, Raza A, Lawhorn Armour BA, Pippin J, Arnerić SP (1990) Immunocytochemical and neurochemical evidence for age-related loss of GABA in the inferior colliculus: implications for neural presbycusis. J Neurosci 10:2363-2372.

Clopton BM, Winfield JA (1973) Tonotopic organization in the inferior colliculus of the rat. Brain Res 56:355-358.

Dallos P (1985) Response characteristics of mammalian cochlear hair cells. J Neurosci 5:1591-1608.

Davies PW, Erulkar SD, Rose JE (1954) Single-unit activity in the auditory cortex of the cat. J Physiol 126:25P.

DeAngelis GC, Ghose GM, Ohzawa I, Freeman RD (1999) Functional micro-organization of primary visual cortex: receptive field analysis of nearby neurons. J Neurosci 19:4046-4064.

DiCarlo JJ, Lane JW, Hsiao SS, Johnson KO (1996) Marking microelectrode penetrations with fluorescent dyes. J Neurosci Methods 64:75-81.

Edwards CJ, Alder TB, Rose GJ (2002) Auditory midbrain neurons that count. Nat Neurosci 5:934-936.

Egorova M, Ehret G, Vartanian I, Esser KH (2001) Frequency response areas of neurons in the mouse inferior colliculus. I. threshold and tuning characteristics. Exp Brain Res 140:145-161.

Ehret G (1974) Age-dependent hearing loss in normal hearing mice. Naturwissenschaften 61:506-507.

Ehret G, Merzenich MM (1988) Complex sound analysis (frequency resolution, filtering and spectral integration) by single units of the inferior colliculus of the cat. Brain Res Rev 13:139-163.

Gittelman JX, Li N, Pollak GD (2009) Mechanisms underlying directional selectivity for frequency-modulated sweeps in the inferior colliculus revealed by in vivo whole-cell recordings. J Neurosci 29:13030-13041.

Hage SR, Ehret G (2003) Mapping responses to frequency sweeps and tones in the inferior colliculus of house mice. Eur J Neurosci 18:2301-2312.

Hamilton LS, Sohl-Dickstein J, Huth AG, Carels VM, Deisseroth K, Bao S (2013) Optogenetic activation of an inhibitory network enhances feedforward functional connectivity in auditory cortex. Neuron 80:10661076.

Hestrin S, Nicoll RA, Perkel DJ, Sah P (1990) Analysis of excitatory synaptic action in pyramidal cells using whole-cell recording from rat hippocampal slices. J Physiol 422:203-225.

Hines M (1993) NEURON: a program for simulation of nerve equations. In: Neural systems: analysis and modeling (Eeckman FH, ed), pp 127-136. Norwell, MA: Kluwer.

Hubel DH, Wiesel TN (1962) Receptive fields, binocular interaction and functional architecture in the cat's visual cortex. J Physiol 160:106-154.

Isaacson JS, Scanziani M (2011) How inhibition shapes cortical activity. Neuron 72:231-243.

Ito T, Bishop DC, Oliver DL (2009) Two classes of GABAergic neurons in the inferior colliculus. J Neurosci 29:13860-13869.

Jahr CE, Stevens CF (1990) Voltage dependence of NMDA-activated macroscopic conductances predicted by single-channel kinetics. J Neurosci 10:3178-3182.

Kaas JH (1989) The evolution of complex sensory systems in mammals. J Exp Biol 146:165-176.

Kato HK, Asinof SK, Isaacson JS (2017) Network-level control of frequency tuning in auditory cortex. Neuron 95:412-423.e4.

Katsuki Y, Watanabe T, Suga N (1959) Interaction of auditory neurons in response to two sound stimuli in cat. J Neurophysiol 22:603-623.

Kiang NY-S, Watanabe T, Thomas EC, Clark LF (1965) Discharge patterns of single fibers in the cat's auditory nerve. Cambridge: MIT.

Koch U, Grothe B (1998) GABAergic and glycinergic inhibition sharpens tuning for frequency modulations in the inferior colliculus of the big brown bat. J Neurophysiol 80:71-82.

Kuo RI, Wu GK (2012) The generation of direction selectivity in the auditory system. Neuron 73:1016-1027.

LeBeau FE, Malmierca MS, Rees A (2001) Iontophoresis in vivo demon- 
strates a key role for $\mathrm{GABA}_{\mathrm{A}}$ and glycinergic inhibition in shaping frequency response areas in the inferior colliculus of guinea pig. J Neurosci 21:7303-7312.

Li H, Liang F, Zhong W, Yan L, Mesik L, Xiao Z, Tao HW, Zhang LI (2018) Synaptic mechanisms for bandwidth tuning in awake mouse primary auditory cortex. Cereb Cortex 29:2998-3009.

Lim HH, Anderson DJ (2007) Spatially distinct functional output regions within the central nucleus of the inferior colliculus: implications for an auditory midbrain implant. J Neurosci 27:8733-8743.

Lu Y, Jen PH (2001) GABAergic and glycinergic neural inhibition in excitatory frequency tuning of bat inferior collicular neurons. Exp Brain Res 141:331-339.

Malmierca MS, Izquierdo MA, Cristaudo S, Hernández O, Pérez -Gonzalez D, Covey E, Oliver DL (2008) A discontinuous tonotopic organization in the inferior colliculus of the rat. J Neurosci 28:4767-4776.

Margrie TW, Brecht M, Sakmann B (2002) In vivo, low-resistance, wholecell recordings from neurons in the anaesthetized and awake mammalian brain. Pflugers Arch 444:491-498.

Mauger SJ, Shivdasani MN, Rathbone GD, Argent RE, Paolini AG (2010) An in vivo investigation of first spike latencies in the inferior colliculus in response to multichannel penetrating auditory brainstem implant stimulation. J Neural Eng 7:036004.

Mayko ZM, Roberts PD, Portfors CV (2012) Inhibition shapes selectivity to vocalizations in the inferior colliculus of awake mice. Front Neural Circuits 6:73.

Moldestad O, Karlsen P, Molden S, Storm JF (2009) Tracheotomy improves experiment success rate in mice during urethane anesthesia and stereotaxic surgery. J Neurosci Methods 176:57-62.

Møller AR (2003) Sensory systems: anatomy and physiology. Amsterdam, Boston: Academic.

Moore LA, Trussell LO (2017) Corelease of inhibitory neurotransmitters in the mouse auditory midbrain. J Neurosci 37:9453-9464.

Nelson S, Toth L, Sheth B, Sur M (1994) Orientation selectivity of cortical neurons during intracellular blockade of inhibition. Science 265:774777.

Ono M, Oliver DL (2014a) The balance of excitatory and inhibitory synaptic inputs for coding sound location. J Neurosci 34:3779-3792.

Ono M, Oliver DL (2014b) Asymmetric temporal interactions of soundevoked excitatory and inhibitory inputs in the mouse auditory midbrain. J Physiol 592:3647-3669.

Ono M, Yanagawa Y, Koyano K (2005) GABAergic neurons in inferior colliculus of the GAD67-GFP knock-in mouse: electrophysiological and morphological properties. Neurosci Res 51:475-492.

Ono M, Bishop DC, Oliver DL (2017) Identified GABAergic and glutamatergic neurons in the mouse inferior colliculus share similar response properties. J Neurosci 37:8952-8964.

Palombi PS, Caspary DM (1996) GABA inputs control discharge rate primarily within frequency receptive fields of inferior colliculus neurons. J Neurophysiol 75:2211-2219.

Peruzzi D, Sivaramakrishnan S, Oliver DL (2000) Identification of cell types in brain slices of the inferior colliculus. Neuroscience 101:403-416.

Pinault D (1996) A novel single-cell staining procedure performed in vivo under electrophysiological control: morpho-functional features of juxtacellularly labeled thalamic cells and other central neurons with biocytin or neurobiotin. J Neurosci Methods 65:113-136.

Poo C, Isaacson JS (2009) Odor representations in olfactory cortex: "sparse" coding, global inhibition, and oscillations. Neuron 62:850-861.

Popper AN, Fay RR (1992) The mammalian auditory pathway: neurophysiology. New York: Springer.

Portfors CV, Wenstrup JJ (2002) Excitatory and facilitatory frequency response areas in the inferior colliculus of the mustached bat. Hear Res 168:131-138

Portfors CV, Mayko ZM, Jonson K, Cha GF, Roberts PD (2011) Spatial organization of receptive fields in the auditory midbrain of awake mouse. Neuroscience 193:429-439.

Priebe NJ, Ferster D (2008) Inhibition, spike threshold, and stimulus selectivity in primary visual cortex. Neuron 57:482-497.

Prosen CA, Dore DJ, May BJ (2003) The functional age of hearing loss in a mouse model of presbycusis: I. Behavioral assessments. Hear Res 183: $44-56$.

Radziwon KE, June KM, Stolzberg DJ, Xu-Friedman MA, Salvi RJ, Dent ML
(2009) Behaviorally measured audiograms and gap detection thresholds in CBA/CaJ mice. J Comp Physiol A Neuroethol Sens Neural Behav Physiol 195:961-969.

Ramachandran R, Davis KA, May BJ (1999) Single-unit responses in the inferior colliculus of decerebrate cats: I. Classification based on frequency response maps. J Neurophysiol 82:152-163.

Russell IJ, Sellick PM (1977) Tuning properties of cochlear hair cells. Nature 267:858-860

Saleem AB, Lien AD, Krumin M, Haider B, Rosón MR, Ayaz A, Reinhold K, Busse L, Carandini M, Harris KD (2017) Subcortical source and modulation of the narrowband gamma oscillation in mouse visual cortex. Neuron 93:315-322.

Shepherd GM, Svoboda K (2005) Laminar and columnar organization of ascending excitatory projections to layer $2 / 3$ pyramidal neurons in rat barrel cortex. J Neurosci 25:5670-5679.

Shu Y, Hasenstaub A, McCormick DA (2003) Turning on and off recurrent balanced cortical activity. Nature 423:288-293.

Somers DC, Nelson SB, Sur M (1995) An emergent model of orientation selectivity in cat visual cortical simple cells. J Neurosci 15:5448-5465.

Spruston N, Jaffe DB, Williams SH, Johnston D (1993) Voltage-clamp and space-clamp errors associated with the measurement of electrotonically remote synaptic events. J Neurophysiol 70:781-802.

Stiebler I, Ehret G (1985) Inferior colliculus of the house mouse: I. A quantitative study of tonotopic organization, frequency representation, and tone-threshold distribution. J Comp Neurol 238:65-76.

Tan AY, Zhang LI, Merzenich MM, Schreiner CE (2004) Tone-evoked excitatory and inhibitory synaptic conductances of primary auditory cortex neurons. J Neurophysiol 92:630-643.

Tan ML, Theeuwes HP, Feenstra L, Borst JG (2007) Membrane properties and firing patterns of inferior colliculus neurons: an in vivo patch-clamp study in rodents. J Neurophysiol 98:443-453.

Tao C, Zhang G, Zhou C, Wang L, Yan S, Tao HW, Zhang LI, Zhou Y, Xiong Y (2017) Diversity in excitation-inhibition mismatch underlies local functional heterogeneity in the rat auditory cortex. Cell Rep 19:521-531.

Wagner T (1994) Intrinsic properties of identified neurones in the central nucleus of mouse inferior colliculus. Neuroreport 6:89-93.

Wehr M, Zador AM (2003) Balanced inhibition underlies tuning and sharpens spike timing in auditory cortex. Nature 426:442-446.

Williams SR, Mitchell SJ (2008) Direct measurement of somatic voltage clamp errors in central neurons. Nat Neurosci 11:790-798.

Willott JF, Urban GP (1978) Response properties of neurons in nuclei of mouse inferior colliculus. J Comp Physiol 127:175-184.

Winer JA, Schreiner CE (2005) The inferior colliculus: with 168 illustrations. New York: Springer.

Woolley SM, Portfors CV (2013) Conserved mechanisms of vocalization coding in mammalian and songbird auditory midbrain. Hear Res 305:45-56.

Wu GK, Li P, Tao HW, Zhang LI (2006) Nonmonotonic synaptic excitation and imbalanced inhibition underlying cortical intensity tuning. Neuron 52:705-715.

Wu GK, Arbuckle R, Liu BH, Tao HW, Zhang LI (2008) Lateral sharpening of cortical frequency tuning by approximately balanced inhibition. Neuron 58:132-143.

Xiong XR, Liang FX, Li HF, Mesik L, Zhang KK, Polley DB, Tao HW, Xiao ZJ, Zhang LI (2013) Interaural level difference-dependent gain control and synaptic scaling underlying binaural computation. Neuron 79:738-753.

Young ED, Brownell WE (1976) Responses to tones and noise of single cells in dorsal cochlear nucleus of unanesthetized cats. J Neurophysiol 39:282-300.

Zhang LI, Tan AY, Schreiner CE, Merzenich MM (2003) Topography and synaptic shaping of direction selectivity in primary auditory cortex. Nature 424:201-205.

Zheng QY, Johnson KR, Erway LC (1999) Assessment of hearing in 80 inbred strains of mice by ABR threshold analyses. Hear Res 130:94-107.

Zhou M, Li YT, Yuan W, Tao HW, Zhang LI (2015) Synaptic mechanisms for generating temporal diversity of auditory representation in the dorsal cochlear nucleus. J Neurophysiol 113:1358-1368.

Zhou Y, Liu BH, Wu GK, Kim YJ, Xiao Z, Tao HW, Zhang LI (2010) Preceding inhibition silences layer 6 neurons in auditory cortex. Neuron 65:706-717. 